\title{
Dynamics of a Pendulum of Variable Length and Similar Problems
}

\author{
A. O. Belyakov and A. P. Seyranian \\ Additional information is available at the end of the chapter
}

http://dx.doi.org/10.5772/48168

\section{Introduction}

In this chapter we study three mechanical problems: dynamics of a pendulum of variable length, rotations of a pendulum with elliptically moving pivot and twirling of a hula-hoop presented in three subsequent sections. The dynamics of these mechanical systems is described by similar equations and is studied with the use of common methods. The material of the chapter is based on publications of the authors [1-7] with the renewed analytical and numerical results. The methodological peculiarity of this work is in the assumption of quasi-linearity of the systems which allows us to derive higher order approximations by the averaging method. All the approximate solutions are compared with the results of numerical simulation demonstrating good agreement. Supplementary, in Appendix (section 5) we briefly presented the method of averaging with higher order approximations which is used in sections 2,3 , and 4 .

\section{Pendulum with periodically variable length}

Oscillations of a pendulum with periodically variable length (PPVL) is the classical problem of mechanics. Usually, the PPVL is associated with a child's swing, see Fig. 1. Everyone can remember that to swing a swing one must crouch when passing through the middle vertical position and straighten up at the extreme positions, i.e. perform oscillations with a frequency which is approximately twice the natural frequency of the swing. Among previous works we cite [8-15] in which analytical and numerical results on dynamic behavior of the PPVL were presented.

The present section is devoted to the study of regular and chaotic motions of the PPVL. Asymptotic expressions for boundaries of instability domains near resonance frequencies are derived. Domains for oscillation, rotation, and oscillation-rotation motions in parameter space are found analytically and compared with numerical study. Chaotic motions of the pendulum depending on problem parameters are investigated numerically. Here we extend our results published in [1-4] in investigating dynamics of this rather simple but interesting mechanical system. 


\subsection{Main relations}

Equation for motion of the swing can be derived with the use of angular momentum alteration theorem, see [8-11]. Taking into account also linear damping forces we obtain

$$
\frac{d}{d t}\left(m l^{2} \frac{d \theta}{d t}\right)+\gamma l^{2} \frac{d \theta}{d t}+m g l \sin \theta=0
$$

where $m$ is the mass, $l$ is the length, $\theta$ is the angle of the pendulum deviation from the vertical position, $g$ is the acceleration due to gravity, and $t$ is the time, Fig. 1 .
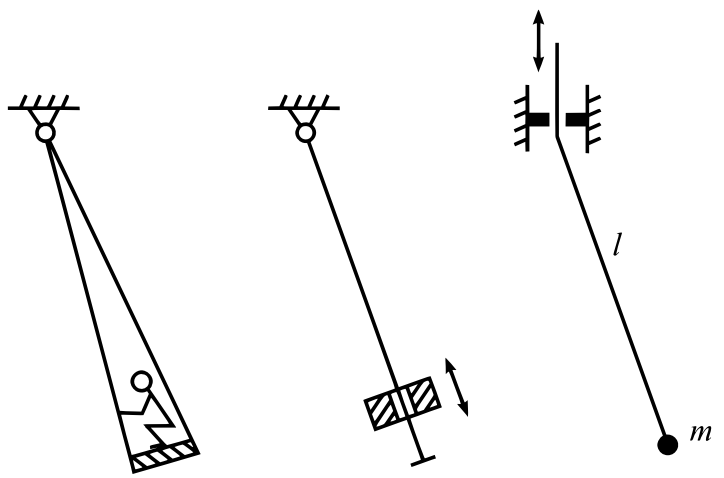

Figure 1. Schemes of the pendulum with periodically varying length.

It is assumed that the length of the pendulum changes according to the periodic law

$$
l=l_{0}+a \varphi(\Omega t)>0,
$$

where $l_{0}$ is the mean pendulum length, $a$ and $\Omega$ are the amplitude and frequency of the excitation, $\varphi(\tau)$ is the smooth periodic function with period $2 \pi$ and zero mean value.

We introduce new time $\tau=\Omega t$ and three dimensionless parameters

$$
\varepsilon=\frac{a}{l_{0}}, \quad \omega=\frac{\Omega_{0}}{\Omega}, \quad \beta=\frac{\gamma}{m \Omega_{0}},
$$

where $\Omega_{0}=\sqrt{\frac{g}{l_{0}}}$ is the eigenfrequency of the pendulum with constant length $l=l_{0}$ and zero damping. In this notations equation (1) takes the form

$$
\ddot{\theta}+\left(\frac{2 \varepsilon \dot{\varphi}(\tau)}{1+\varepsilon \varphi(\tau)}+\beta \omega\right) \dot{\theta}+\frac{\omega^{2} \sin (\theta)}{1+\varepsilon \varphi(\tau)}=0,
$$

where the upper dot denotes differentiation with respect to new time $\tau$.

Stability and oscillations of the system governed by equation (4) will be studied in the following subsections via analytically under the assumption that the excitation amplitude $\varepsilon$ and the damping coefficient $\varepsilon$ are small. For rotational orbits we will also assume the smallness of the frequency $\omega$ which means high excitation frequency compared with the eigenfrequency $\Omega_{0}$. 


\subsection{Instability of the vertical position}

It is convenient to change the variable by the substitution

$$
\eta=\theta(1+\varepsilon \varphi(\tau)) .
$$

Using this substitution in equation (4) and multiplying it by $1+\varepsilon \varphi(\tau)$ we obtain the equation for $\eta$ as

$$
\ddot{\eta}+\beta \omega \dot{\eta}-\frac{\varepsilon(\ddot{\varphi}(\tau)+\beta \omega \dot{\varphi}(\tau))}{1+\varepsilon \varphi(\tau)} \eta+\omega^{2} \sin \left(\frac{\eta}{1+\varepsilon \varphi(\tau)}\right)=0
$$

This equation is useful for stability study of the vertical position of the pendulum as well as analysis of small oscillations.

Let us analyze the stability of the trivial solution $\eta=0$ of the nonlinear equation (6). Its stability with respect to the variable $\eta$ is equivalent to that of the equation (4) with respect to $\theta$ due to relation (5). According to Lyapunov's theorem on stability based on a linear approximation for a system with periodic coefficients the stability (instability) of the solution $\eta=0$ of equation (6) is determined by the stability (instability) of the linearized equation

$$
\ddot{\eta}+\beta \omega \dot{\eta}+\frac{\omega^{2}-\varepsilon(\ddot{\varphi}(\tau)+\beta \omega \dot{\varphi}(\tau))}{1+\varepsilon \varphi(\tau)} \eta=0 .
$$

This equation explicitly depends on three parameters: $\varepsilon, \beta$ and $\omega$. Expanding the ratio in (7) into Taylor series and keeping only first order terms with respect to $\varepsilon$ and $\beta$ we obtain

$$
\ddot{\eta}+\beta \omega \dot{\eta}+\left[\omega^{2}-\varepsilon\left(\ddot{\varphi}(\tau)+\omega^{2} \varphi(\tau)\right)\right] \eta=0 .
$$

This is a Hill's equation with damping with the periodic function $-\left(\ddot{\varphi}(\tau)+\omega^{2} \varphi(\tau)\right)$. It is known that instability (i.e. parametric resonance) occurs near the frequencies $\omega=k / 2$, where $k=1,2, \ldots$. Instability domains in the vicinity of these frequencies were obtained in [16, 17] analytically. In three-dimensional space of the parameters $\varepsilon, \beta$ and $\omega$, these domains are described by half-cones

$$
(\beta / 2)^{2}+(2 \omega / k-1)^{2}<r_{k}^{2} \varepsilon^{2}, \quad \beta \geq 0, \quad k=1,2, \ldots,
$$

where $r_{k}=\frac{3}{4} \sqrt{a_{k}^{2}+b_{k}^{2}}$ is expressed through the Fourier coefficients of the periodic function $\varphi(\tau)$

$$
a_{k}=\frac{1}{\pi} \int_{0}^{2 \pi} \varphi(\tau) \cos (k \tau) d \tau, \quad b_{k}=\frac{1}{\pi} \int_{0}^{2 \pi} \varphi(\tau) \sin (k \tau) d \tau .
$$

Inequalities (9) give us the first approximation of the instability domains of the vertical position of the swing. These inequalities were obtained in [1] using different variables.

Note that each $k$-th resonant domain in relations (9) depends only on $k$-th Fourier coefficients of the periodic excitation function. Particularly, for $\varphi(\tau)=\cos (\tau), k=1$ we obtain $a_{1}=1$, $b_{1}=0$, and $r_{1}=3 / 4$. Thus, the first instability domain takes the form

$$
\beta^{2} / 4+(2 \omega-1)^{2}<9 \varepsilon^{2} / 16, \quad \beta \geq 0 .
$$



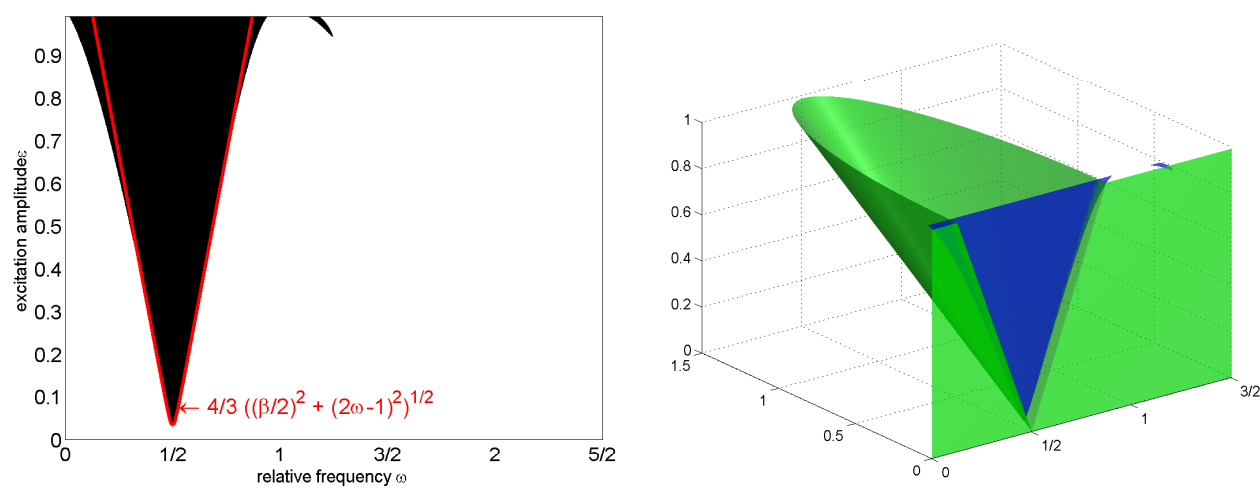

Figure 2. Instability domain (9) of PPVL (red line, left) in comparison with numerical results (black area) on parameter plane $(\omega, \varepsilon)$ at $\beta=0.05$. Half cone instability domain (9) of PPVL (green surface, right) compared with the same numerical results (blue plane) in the parameter space $(\omega, \beta, \varepsilon)$.

The boundary of the first instability domain $(k=1)$ is presented in Fig. 2 by the solid red line demonstrating a good agreement with the numerically obtained instability domain which is marked black. These boundaries are also drawn in Fig. 5 and 6 by solid white lines. It is easy to see from (8) that for the second resonance domain $(k=2, \omega=1)$ the excitation function $-\left(\ddot{\varphi}(\tau)+\omega^{2} \varphi(\tau)\right)$ is zero for $\varphi(\tau)=\cos (\tau)$. This explains why the second resonance domain is empty, and the numerical results confirm this conclusion, see Fig. 2. Inside the instability domains (9) the vertical position $\eta=0$ becomes unstable and motion of the system can be either regular (limit cycle, regular rotation) or chaotic.

\subsection{Limit cycle}

When the excitation amplitude $\varepsilon$ is small, we can expect that the oscillation amplitude $\theta$ in equation (4) will be also small. We suppose that $\varepsilon$ and $\beta$ are small parameters of the same order, and $\theta=O(\sqrt{\varepsilon})$. Then, we introduce notation $\tilde{\beta}=\beta / \varepsilon, \vartheta=\theta / \sqrt{\varepsilon}$ and expand the sine into Taylor's series around zero in equation (4) keeping only three terms. Thus, equation (4) takes the following form

$$
\ddot{\vartheta}+\omega^{2} \vartheta=\varepsilon f_{1}(\vartheta, \dot{\vartheta}, \tau)+\varepsilon^{2} f_{2}(\vartheta, \dot{\vartheta}, \tau)+\ldots,
$$

where

$$
\begin{aligned}
& f_{1}(\vartheta, \dot{\vartheta}, \tau)=\omega^{2}\left(\varphi(\tau) \vartheta+\frac{\vartheta^{3}}{6}\right)-(\tilde{\beta} \omega+2 \dot{\varphi}(\tau)) \dot{\vartheta}, \\
& f_{2}(\vartheta, \dot{\vartheta}, \tau)=2 \dot{\varphi}(\tau) \varphi(\tau) \dot{\vartheta}-\omega^{2}\left(\varphi(\tau)\left(\varphi(\tau) \vartheta+\frac{\vartheta^{3}}{6}\right)+\frac{\vartheta^{5}}{120}\right) .
\end{aligned}
$$

We study the parametric excitation of nonlinear system (12) with the periodic function $\varphi(\tau)=$ $\cos \tau$ at the first resonance frequency, $\omega-1 / 2=O(\varepsilon)$. To solve equation (12) we use the method of averaging [9, 18-20]. For that purpose we write (12) in the Bogolubov's standard form of first order differential equations with small right-hand sides. First, we use Poincaré 
variables $q(\tau)$ and $\psi(\tau)$ defined via the following solution of the generating equation $\ddot{\vartheta}+\omega^{2} \vartheta=$ 0 which is equation (12) with $\varepsilon=0$, when $\dot{q}=0$ and $\dot{\psi}=\omega$

$$
\vartheta=q \cos (\psi), \quad \dot{\vartheta}=-\omega q \sin (\psi) .
$$

We can express Poincaré variables $q(\tau)$ and $\psi(\tau)$ via $\vartheta$ and $\dot{\vartheta}$ from (15) as $q^{2}=\vartheta^{2}+\dot{\vartheta}^{2} / \omega^{2}$ and $\psi=\arctan \left(-\frac{\dot{\theta}}{\vartheta \omega}\right)$. We differentiate these equations with respect to time and substitute expressions for $\ddot{\vartheta}, \dot{\vartheta}$, and $\vartheta$ in terms of $q$ and $\psi$ obtained from (12), and (15). Then, in the resonant case we have equations for the slow amplitude $q(\tau)$ and phase shift $\zeta(\tau)=\psi(\tau)-\frac{1}{2} \tau$

$$
\begin{aligned}
& \dot{q}=-\frac{\sin \left(\frac{\tau}{2}+\zeta\right)}{\omega} f\left(q \cos \left(\frac{\tau}{2}+\zeta\right),-q \omega \sin \left(\frac{\tau}{2}+\zeta\right), \tau\right), \\
& \dot{\zeta}=\omega-\frac{1}{2}-\frac{\cos \left(\frac{\tau}{2}+\zeta\right)}{\omega q} f\left(q \cos \left(\frac{\tau}{2}+\zeta\right),-q \omega \sin \left(\frac{\tau}{2}+\zeta\right), \tau\right),
\end{aligned}
$$

where $f(\vartheta, \dot{\vartheta}, \tau)=\varepsilon f_{1}(\vartheta, \dot{\vartheta}, \tau)+\varepsilon^{2} f_{2}(\vartheta, \dot{\vartheta}, \tau)+o\left(\varepsilon^{2}\right)$. System (16)-(17) has small right hand sides because we assumed that $\omega-1 / 2=O(\varepsilon)$. As a result of averaging in the second approximation, see (121) in the Appendix, we get the system of averaged differential equations

$$
\begin{aligned}
\dot{Q} & =\varepsilon Q\left(\frac{2-\omega}{4} \sin (2 Z)-\frac{\tilde{\beta} \omega}{2}\right)+\varepsilon^{2} Q \omega\left(5 Q^{2} \frac{2-\omega}{192} \sin (2 Z)+\frac{\tilde{\beta} \omega}{4} \cos (2 Z)\right)+o\left(\varepsilon^{2}\right), \\
\dot{Z} & =\omega-\frac{1}{2}+\varepsilon\left(\frac{2-\omega}{4} \cos (2 Z)-\frac{Q^{2} \omega}{16}\right) \\
& +\varepsilon^{2}\left(5 Q^{2} \omega \frac{2-\omega}{96} \cos (2 Z)-\frac{\tilde{\beta} \omega^{2}}{4} \sin (2 Z)-Q^{4} \omega \frac{17 \omega-4}{1536}-\frac{(2-\omega)^{2}}{32}-\frac{\omega^{2} \tilde{\beta}^{2}}{4}\right)+o\left(\varepsilon^{2}\right),
\end{aligned}
$$

where $Q$ and $Z$ are the averaged variables corresponding to $q$ and $\zeta$. This system gives steady solutions for $\dot{Q}=0, \dot{Z}=0$. Thus, besides the trivial one $Q=0$, in the first approximation we obtain from system (18)-(19) expressions for the averaged amplitude and phase shift as

$$
\begin{aligned}
& \varepsilon Q_{\{1\}}^{2}=\frac{4}{\omega}\left(4 \omega-2 \mp \sqrt{\varepsilon^{2}(2-\omega)^{2}-4 \beta^{2} \omega^{2}}\right), \\
& Z_{\{1\}}=\frac{1}{2} \arctan \left(\frac{\mp 2 \beta \omega}{\sqrt{\varepsilon^{2}(2-\omega)^{2}-4 \beta^{2} \omega^{2}}}\right)+\pi j,
\end{aligned}
$$

where $j=\ldots,-1,0,1,2, \ldots$ and "arctan" gives the major function value lying between zero and $\pi$; subindex " $\{1\}$ " denotes the order of approximation with which the corresponding variable is obtained. Solution of system (16)-(17) in the first approximation is $q=Q_{\{1\}}+o(1)$, $\zeta=Z_{\{1\}}+o(1)$ so the solution of (4) is $\theta=\sqrt{\varepsilon} Q_{\{1\}} \cos \left(\tau / 2+Z_{\{1\}}\right)+o(\sqrt{\varepsilon})$.

Solution of system (16)-(17) in the second approximation is the following, see (119) and (121),

$$
\begin{aligned}
q= & Q_{\{2\}}+\varepsilon Q_{\{2\}}\left(\frac{\tilde{\beta} \omega}{2} \sin \left(\tau+2 Z_{\{2\}}\right)-\cos (\tau)+\frac{\omega+2}{8} \cos \left(2 \tau+2 Z_{2}\right)\right) \\
& +\varepsilon Q_{\{2\}}^{3}\left(\frac{\omega}{24} \cos \left(\tau+2 Z_{\{2\}}\right)+\frac{\omega}{96} \cos \left(2 \tau+4 Z_{\{2\}}\right)\right)+o(\varepsilon), \\
\zeta= & Z_{\{2\}}+\varepsilon\left(\frac{\tilde{\beta} \omega}{2} \cos \left(\tau+2 Z_{\{2\}}\right)-\frac{\omega}{2} \sin (\tau)-\frac{\omega+2}{8} \sin \left(2 \tau+2 Z_{\{2\}}\right)\right) \\
& -\varepsilon Q_{\{2\}}^{2}\left(\frac{\omega}{12} \sin \left(\tau+2 Z_{\{2\}}\right)+\frac{\omega}{96} \sin \left(2 \tau+4 Z_{\{2\}}\right)\right)+o(\varepsilon),
\end{aligned}
$$


where $Q_{\{2\}}$ and $Z_{\{2\}}$ are the steady state variables of system (18)-(19) in the second order approximation. Substitution of these expressions into (15) yields the second order approximate solution of (4) in the following form

$$
\begin{aligned}
\theta & =\sqrt{\varepsilon} Q_{\{2\}} \cos \left(\frac{\tau}{2}+Z_{\{2\}}\right)+\beta \sqrt{\varepsilon} Q_{\{2\}} \frac{\omega}{2} \sin \left(\frac{\tau}{2}+Z_{\{2\}}\right) \\
& -\varepsilon^{\frac{3}{2}} Q_{\{2\}}\left(\frac{2-\omega}{4} \cos \left(\frac{\tau}{2}-Z_{\{2\}}\right)+\frac{2+\omega}{8} \cos \left(\frac{3 \tau}{2}+Z_{\{2\}}\right)\right) \\
& +\varepsilon^{\frac{3}{2}} Q_{\{2\}}^{3}\left(\frac{\omega}{16} \cos \left(\frac{\tau}{2}+Z_{\{2\}}\right)-\frac{\omega}{96} \cos \left(\frac{3 \tau}{2}+3 Z_{\{2\}}\right)\right)+o\left(\varepsilon^{2}\right) .
\end{aligned}
$$
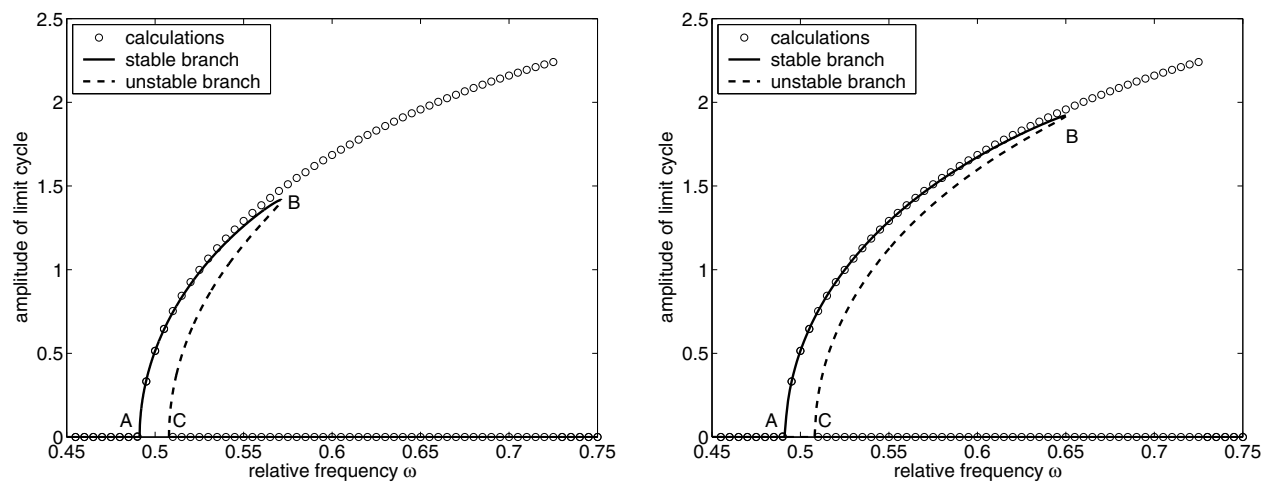

Figure 3. Frequency-response curve for the parameters $\varepsilon=0.04$ and $\beta=0.05$. Amplitude of the limit cycle in the first (left) and second (right) approximation compared with the results of numerical simulations (circles) depending on the relative excitation frequency $\omega$. In the first approximation (left) the amplitude $\sqrt{\varepsilon} Q_{\{1\}}$ is described by (20). In the second approximation (right) the amplitude of solution (24) is calculated with the use of numerically obtained steady state $Q_{\{2\}}$ and $Z_{\{2\}}$ of system (18)-(19).

Fig. 3 shows better coincidence with the numerical simulations of the second order approximate solution (24) up to the amplitude equals $2 \approx 2 \pi / 3$ and the frequency mismatch is $\omega-\frac{1}{2} \approx 0.15$.

\subsection{Regular rotations}

We say that the system performs regular rotations if a nonzero average rotational velocity exists

$$
b=\lim _{T \rightarrow \infty} \frac{1}{T} \int_{0}^{T} \dot{\theta} d \tau .
$$

Velocity $b$ is a rational number because regular motions can be observed only in resonance with excitation. Motion with fractional average velocity such as $|b|=1 / 2$ in Fig. 4 a) is usually called oscillation-rotation. Let us first study monotone rotations, where velocity $\dot{\theta}$ has constant sign and integer average value $b$, see Fig. 4 b) and c).

In order to describe resonance rotations of the PPVL we use the method of averaging $[9,18,19]$ which requires rewriting (4) in the Bogolubov's standard form. For that reason we assume 


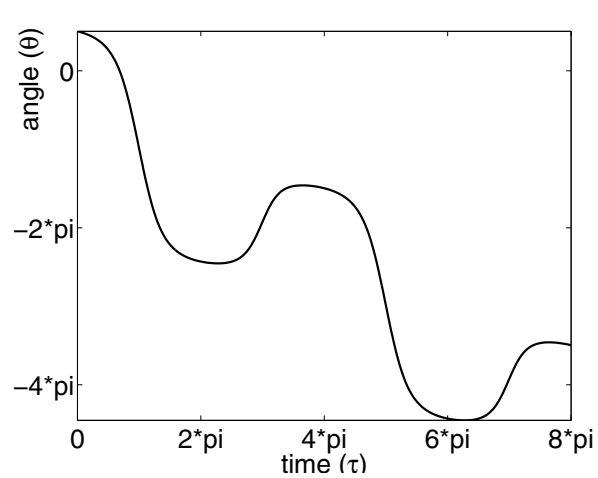

a) $\varepsilon=0.51, \omega=0.54, \beta=0.05$

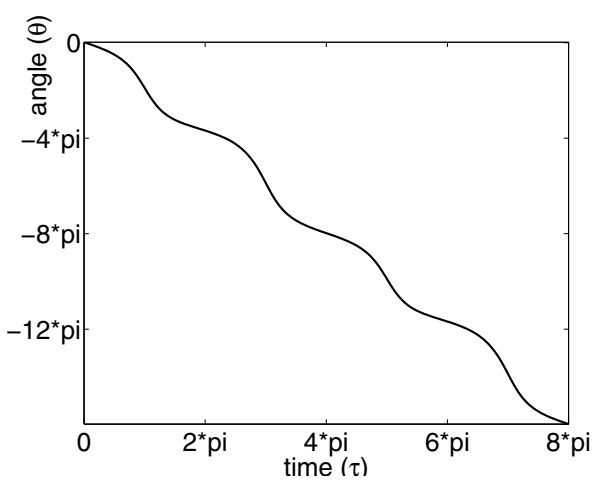

c) $\varepsilon=0.43, \omega=0.5, \beta=0.05$

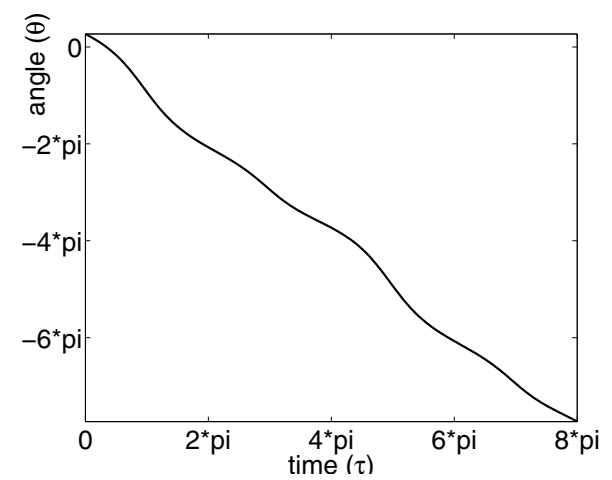

b) $\varepsilon=0.28, \omega=0.5, \beta=0.05$

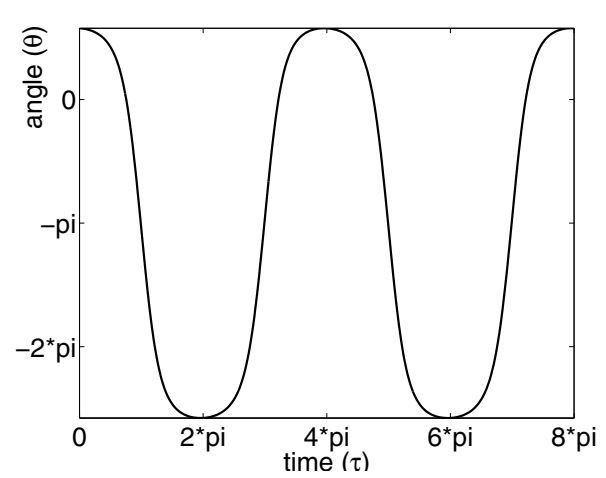

d) $\varepsilon=0.59, \omega=0.6, \beta=0.05$

Figure 4. a) Regular rotation-oscillation with the mean angular velocity equal to one half of the excitation frequency, $b=-1 / 2$. b) Regular rotation with $b=-1$. c) Regular rotation with $b=-2$. d) Regular rotation-oscillation with $b=0$.

that $\varepsilon, \beta$ and $\omega$ are small parameters, $\varepsilon$ being of order $\omega^{2}$, and $\beta$ of order $\omega^{3}$, which makes the system quasi-linear.

We introduce the vector of slow variables $x$ and the fast time $s=|b| \tau$, where $x_{1}=\theta-b \tau$ is the phase mismatch, $x_{2}=\frac{d \theta}{d s}$ is the velocity, $x_{3}=1+\varepsilon \cos \left(\frac{s}{|b|}\right)$ is the excitation. From here the dot denotes derivative with respect to the new time $s$. Thus, equations (4) takes the standard form

$$
\begin{aligned}
& \dot{x}_{1}=x_{2}-\operatorname{sign}(b), \\
& \dot{x}_{2}=\left(\frac{2 \varepsilon}{x_{3}} \sin \left(\frac{s}{|b|}\right)-\beta \omega\right) \frac{x_{2}}{|b|}-\frac{\omega^{2}}{b^{2}} \frac{\sin \left(x_{1}+s\right)}{x_{3}}, \\
& \dot{x}_{3}=-\frac{\varepsilon}{|b|} \sin \left(\frac{s}{|b|}\right),
\end{aligned}
$$


where it is assumed that $x_{2}-\operatorname{sign}(b)$ is of order $\varepsilon, \operatorname{sign}(b)=1$ if $b>0$ and $\operatorname{sign}(b)=-1$ if $b<0$. With the method of averaging we can find the first, second and the following order approximations of equations (25).

Resonance rotation domains of PPVL for various $|b|$ are presented in Fig. 5. We see that greater values of relative rotational velocities $|b|$ are possible for higher excitation amplitudes $\varepsilon$. Numerically obtained rotational regimes are depicted in Fig. 5 by color points in parameter space $(\omega, \varepsilon)$ with $\beta=0.05$ and initial conditions $\theta(0)=\pi, \dot{\theta}(0)=0.05$. Domains of these points are well bounded below by analytically obtained curves for corresponding $|b|$.

\subsubsection{Rotations with relative velocity $|b|=1$}

It is the third order approximation of averaged equation where regular rotations with $|b|=1$ can be observed, see Fig. 4 b). In the third order approximation averaged equations take the following form, see (122),

$$
\begin{aligned}
& \dot{X}_{1}=X_{2}-b, \\
& \dot{X}_{2}=-\frac{3 \varepsilon \omega^{2}}{2} \sin \left(X_{1}\right)-\beta \omega X_{2},
\end{aligned}
$$

where $X_{1}$ and $X_{2}$ are the averaged slow variables $x_{1}$ and $x_{2}$. Auxiliary variable $x_{3}=1+$ $\varepsilon \cos (s / b)$ has unit average $X_{3}=1$ and is excluded from the consideration. Excluding variable $X_{2}$ from the steady state conditions $\dot{X}_{1}=0$ and $\dot{X}_{2}=0$ in (26) we obtain the equation for the averaged phase mismatch $X_{1}$

$$
\sin \left(X_{1}\right)=-b \frac{2 \beta}{3 \varepsilon \omega} .
$$

Thus, it is clear from (27) that equation (26) has a steady state solution only if

$$
\omega \geq \frac{2 \beta}{3 \varepsilon} \text {. }
$$

Inequality (28) determines the domain in parameter space, where rotations with $|b|=1$ can exist. The boundary of this domain is depicted with a bold dashed line in Fig. 5 on the parameter plane $(\omega, \varepsilon)$ for $\beta=0.05$.

Stability of the solutions obtained from (27) was studied in [2]. There was found the condition for asymptotic stability $\cos \left(X_{1}\right)>0$. Hence, if inequality (28) is strict, then there are asymptotically stable steady solutions

$$
X_{1(1)}=-b \arcsin \left(\frac{2 \beta}{3 \omega \varepsilon}\right)+2 \pi k, \quad k=\ldots,-1,0,1,2, \ldots
$$

and unstable solutions

$$
X_{1(2)}=\pi+b \arcsin \left(\frac{2 \beta}{3 \omega \varepsilon}\right)+2 \pi k, \quad k=\ldots,-1,0,1,2, \ldots
$$

Thus, we conclude that if the parameters satisfy strict inequality (28) there are two stable regular rotations $\theta=b \tau+X_{1(1)}+o(1)$ in opposite directions $(b= \pm 1)$ and two unstable rotations $\theta=b \tau+X_{1(2)}+o(1)$ in opposite directions. 


\subsubsection{Rotations with relative velocity $|b|=2$}

Rotations with higher averaged velocities $|b|=2, \ldots$ correspond to higher excitation amplitudes $\varepsilon$. That is why we consider the coefficient $\omega$ being of order $\varepsilon$, and $\beta$ being of order $\varepsilon^{3}$. With this new ordering we obtain the sixth order approximation of the averaged equations for $|b|=2$, see (128),

$$
\begin{aligned}
& \dot{X}_{1}=X_{2}-\frac{b}{2} \\
& \dot{X}_{2}=-\frac{9 \varepsilon^{2} \omega^{2}}{16}\left(1-\left(X_{2}-\frac{b}{2}\right)^{2}+\frac{\varepsilon^{2}}{27}\right) \sin \left(X_{1}\right)-\frac{\beta \omega}{2} X_{2},
\end{aligned}
$$

which have steady state solutions determined by the following equation

$$
\sin \left(X_{1}\right)=-b \frac{8 \beta}{9 \varepsilon^{2} \omega}\left(\frac{1}{1+\varepsilon^{2} / 27}\right) .
$$

From equation (32) we get that the domain of rotations with $|b|=2$ in the parameter space has the following boundary condition depicted in Fig. 5 with a bold solid line

$$
\omega \geq \frac{8 \beta}{9 \varepsilon^{2}}\left(\frac{1}{1+\varepsilon^{2} / 27}\right) .
$$

System (31) has similar structure to system (26). That is why stability condition for its steady state solutions appears to be the same: $\cos \left(X_{1}\right)>0$. Hence, if inequality (33) is strict, we find from (32) that there are asymptotically stable steady solutions

$$
X_{1(1)}=-\arcsin \left(\frac{8 b \beta}{9 \varepsilon^{2} \omega}\left(\frac{1}{1+\varepsilon^{2} / 27}\right)\right)+2 \pi k, \quad k=\ldots,-1,0,1,2, \ldots
$$

and unstable steady solutions

$$
X_{1(2)}=\pi+\arcsin \left(\frac{8 b \beta}{9 \varepsilon^{2} \omega}\left(\frac{1}{1+\varepsilon^{2} / 27}\right)\right)+2 \pi k, \quad k=\ldots,-1,0,1,2, \ldots
$$

Thus, as in the previous case, if the parameters satisfy strict inequality (33) there are two stable regular rotations $\theta=b \tau+X_{1(1)}+o(1)$ in opposite directions $(b= \pm 2)$ and two unstable rotations $\theta=b \tau+X_{1(2)}+o(1)$ in opposite directions.

\subsection{Basins of attractions and transitions to chaos}

In order to determine domains of chaos we calculate maximal Lyapunov exponents presented in Fig. 6. We recall that positive Lyapunov exponents correspond to chaotic motions. Note that chaotic motion includes passing through the upper vertical position, i.e. irregular oscillations-rotations. This is usually called tumbling chaos. We have observed two types of transition to chaos. The first type is when the system goes through the cascade of period doubling (PD) bifurcations occurring within the instability domain of the vertical position when the excitation amplitude $\varepsilon$ increases, for example at $\omega=0.5$ in Fig. 7(a). The second type is when chaos immediately appears after subcritical Andronov-Hopf (AH) bifurcation when the system enters the instability domain of the lower vertical position of PPVL, for 


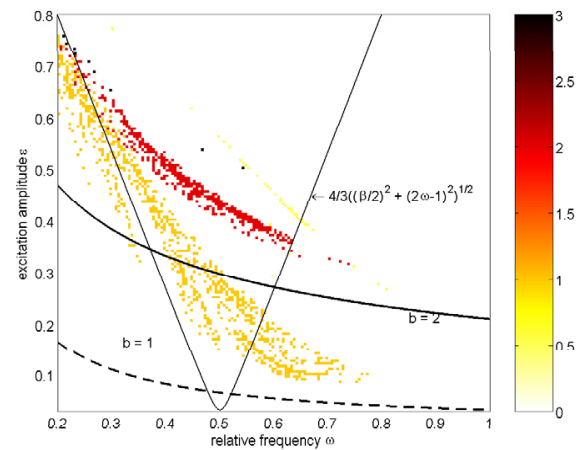

Figure 5. Absolute values $|b|$ of relative rotational velocities are shown with different colors on the plane of parameters $\varepsilon$ and $\omega$ at the damping $\beta=0.05$. The correspondence between the colors and values is shown by the color bar on the right. Approximate boundaries for rotations are drawn with bold dashed line (for $|b|=1$ ) and bold solid line (for $|b|=2$ ).

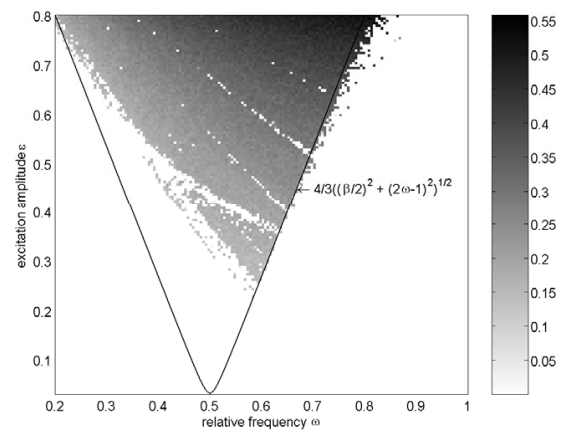

Figure 6. Maximal Lyapunov's exponents are shown on the plane of parameters $\varepsilon$ and $\omega$ at the damping $\beta=0.05$. The correspondence between the colors and values is shown by the color bar on the right, where white color distinguishes zero maximal Lyapunov's exponent which corresponds to regular regime. Positive Lyapunovs' exponents characterize chaotic motions.

example at $\omega=0.67$; see Fig. 7(b). We can see the change of the system dynamics in its route to chaos along $\omega=0.5$ in the bifurcation diagram shown in Fig. 7(a), where red points denote rotations with mean angular velocity equal to one excitation frequency $(|b|=1)$ and green points denote those equal to two excitation frequencies $(|b|=2)$. The domain with the most complex regular dynamics is surrounded by the red rectangle, where the system can have coexisting oscillations, rotations and rotations-oscillations.

Basins of attractions in Fig. 8 have been plotted using program Dynamics [21]. These basins track the changes of the system dynamics in its route to chaos along $\omega=0.67$. In Fig. 8(a) the oscillatory attractor (limit cycle) coexists with stationary attractor (lower vertical position of PPVL). In Fig. 8(b) we can see the first emergence of two rotational attractors with counterrotations. This picture is in a good agreement with condition (18) for existence of rotational solutions $|b|=1$, see Fig. 5. Closer to the boundary of chaotic region in Fig. 8(c) only stationary and rotational attractors remain. Note that the basins of rotational attractors are 


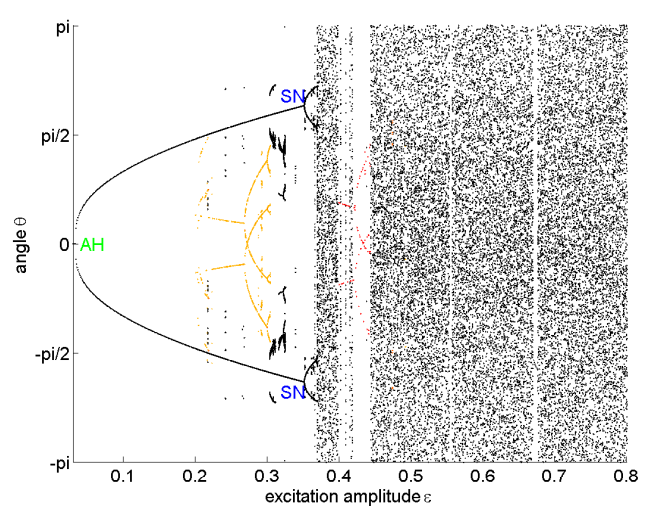

(a) $\omega=0.5$

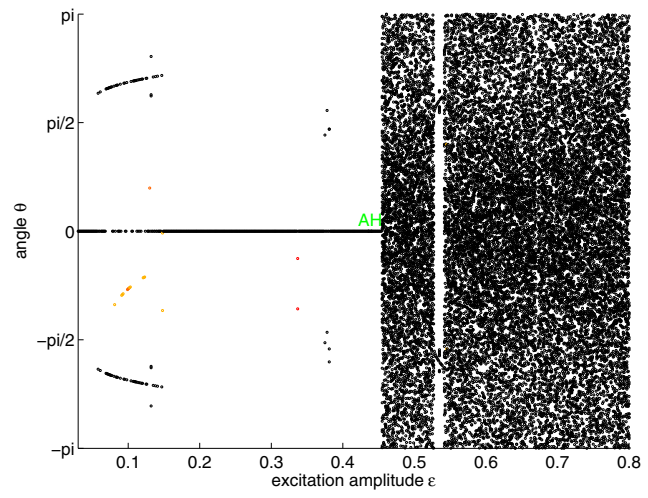

(b) $\omega=0.67$

Figure 7. The bifurcation diagrams for different frequencies $\omega$ and the same damping $\beta=0.05$ show two different types of transition to chaos. (a) After Andronov-Hopf bifurcation (AH) a limit cycle appears which experiences the saddle-node bifurcation (SN) and then the cascade of period-doubling bifurcations (PD). Regular rotations with relative mean angular velocity $|b|=1$ are denoted by orange points and $|b|=2$ by red. (b) After subcritical AH bifurcation of the vertical equilibrium the chaotic motion occurs immediately.

small which means that at $\omega=0.67$ the transition to chaos through subcritical AH bifurcation is the most typical. In the middle of Fig. 8(d) the manifold of dark blue points reveals a typical strange attractor structure. The strange attractor inherits the basin of attraction from disappeared stationary attractor.

\section{Elliptically excited pendulum}

Elliptically excited pendulum (EEP) is a mathematical pendulum in the vertical plane whose pivot oscillates not only vertically but also horizontally with $\pi / 2$ phase shift, so that the pivot has elliptical trajectory, see Fig. 9. EEP is a natural generalization of pendulum with vertically vibrating pivot that is one of the most studied classical systems with parametric excitation. It is often referred to simply as parametric pendulum, see e.g. [9, 22-27] and references therein. Stability and dynamics of EEP have been studied analytically and numerically in [28-30]. Approximate oscillatory and rotational solutions for EEP are the common examples in literature [31-34] on asymptotic methods. Sometimes EEP is presented in a slightly more general model of unbalanced rotor [31-33], where the phase shift between vertical and horizontal oscillations of the pivot can differ from $\pi / 2$. EEP is also a special case of generally excited pendulum in [35]. The usual assumption for approximate solution in the literature is the smallness of dimensionless damping and pivot oscillation amplitudes in the EEP's equation of motion. We could find only one paper [36], where oscillations of EEP with high damping and yet small relative excitation were studied.

In this section we study rotations of EEP with not small excitation amplitudes and with both small and not small linear damping. Our analysis uses the exact solutions for EEP with the absence of gravity and with equal excitation amplitudes, when elliptical trajectory of the pivot 

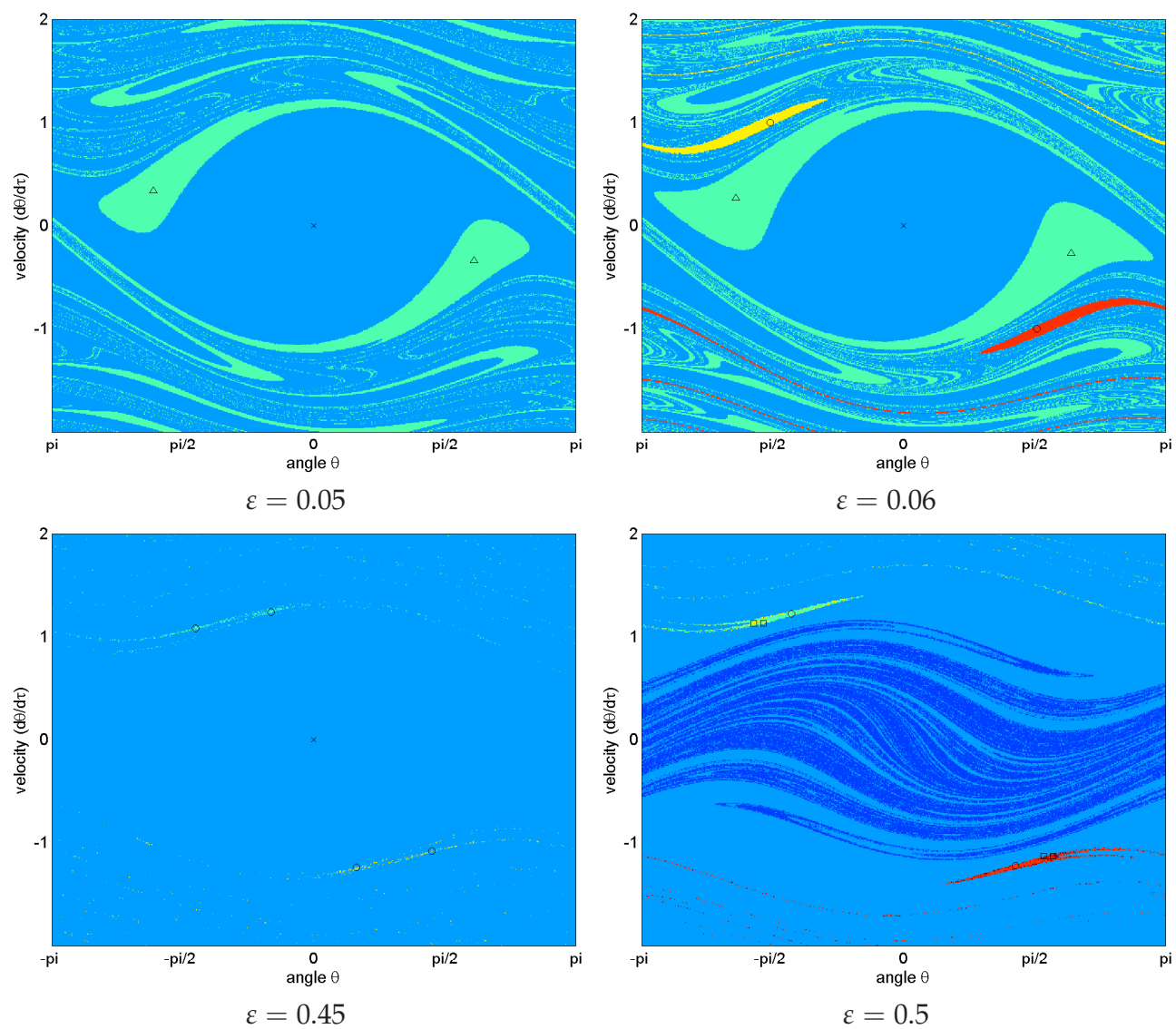

Figure 8. Basins of attractions in Poincare section for different excitation amplitude $\varepsilon$ at the same frequency $\omega=0.67$ and damping $\beta=0.05$. $\triangle$ marks period-two oscillational attractors, $\bigcirc$ marks period-one rotational attractors, $\square$ marks period-two rotational attractors, $\times$ marks fixed points.

becomes circular. When there is no gravity the model of EEP coincides with that of hula-hoop, see section 4 . The material of the section is based on the paper [5].

\subsection{Main relations}

Equation of EEP's motion can be derived with the use of angular momentum alteration theorem, see [30]

$$
\begin{aligned}
m l^{2} \frac{d^{2} \theta}{d t^{2}}+c \frac{d \theta}{d t} & +m l\left(g \cos (\delta)-\frac{d^{2} y(t)}{d t^{2}}\right) \sin (\theta) \\
& +m l\left(g \sin (\delta)-\frac{d^{2} x(t)}{d t^{2}}\right) \cos (\theta)=0,
\end{aligned}
$$




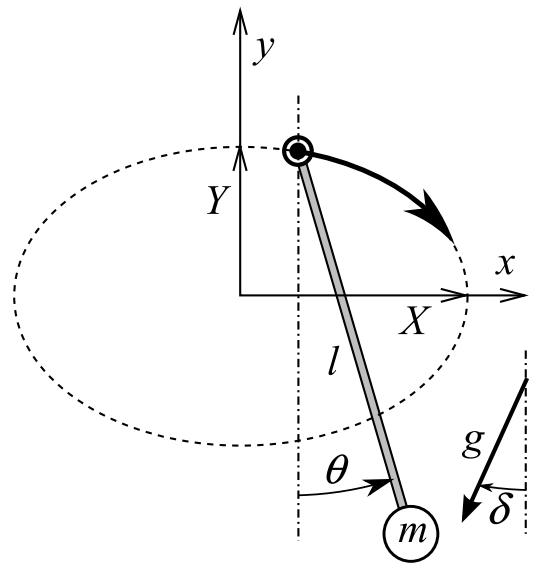

Figure 9. Scheme of the elliptically excited mathematical pendulum of length $l$. The pivot of the pendulum moves along the elliptic trajectory (dashed line) with semiaxes $X$ and $Y$ in the uniform gravitational field $g$.

where $l$ is the distance between the pivot and the concentrated mass $m ; c$ is the viscous damping coefficient; $\theta$ is the angle of the pendulum deviation from the vertical position; $t$ is time; $g$ is gravitational acceleration at the angle $\delta$ with respect to the negative direction of the axis $y$.

It is assumed that the pivot of the pendulum moves according to the periodic law

$$
x=X \sin (\Omega t), \quad y=Y \cos (\Omega t),
$$

where $X, Y$, and $\Omega$ are the amplitudes and frequency of the excitation.

We introduce new time $\tau=\Omega t$ and the following dimensionless parameters

$$
\varepsilon=\frac{Y-X}{2 l}, \quad \mu=\frac{Y+X}{2 l}>0, \quad \omega=\frac{1}{\Omega} \sqrt{\frac{g}{l}}, \quad \beta=\frac{c}{m l^{2} \Omega} .
$$

With this notation equation (36) with substituted (37) in it takes the following form

$$
\ddot{\theta}+\beta \dot{\theta}+\mu \sin (\tau+\theta)=\varepsilon \sin (\tau-\theta)-\omega^{2} \sin (\theta+\delta),
$$

where we use the formula $Y \cos (\Omega t) \sin (\theta)+X \sin (\Omega t) \cos (\theta)=\frac{Y+X}{2} \sin (\Omega t+\theta)-$ $\frac{Y-X}{2} \sin (\Omega t-\theta) .{ }^{1}$ Here the upper dot denotes differentiation with respect to the new time $\tau$.

\subsection{Exact rotational solution when $\varepsilon=0$ and $\omega=0$}

Conditions $\varepsilon=\omega=0$ mean that we find the mode of rotation for the circular excitation $X=Y$ with absence of gravity $g=0$. In this case, we call equation (39) the unperturbed equation

\footnotetext{
${ }^{1}$ Note that this formula excludes the generalization $y=Y \cos (\Omega t+\Phi)$ which is considered e.g. in the model of unbalanced rotor [31-33]. Instead of $\Phi$ we introduce the angle $\delta$ of deviation of gravitational acceleration $g$ from the vertical direction.
} 


$$
\ddot{\theta}+\beta \dot{\theta}+\mu \sin (\tau+\theta)=0
$$

which has exact solutions

$$
\theta=\theta_{0}-\tau
$$

where constants $\theta_{0}$ are defined by the following equality

$$
\sin \left(\theta_{0}\right)=\frac{\beta}{\mu}
$$

provided that $|\beta| \leq \mu$.

To investigate the stability of these solutions we present the angle $\theta$ as $\theta=\theta_{0}-\tau+\eta$, where $\eta=\eta(\tau)$ is a small addition, and substitute it in equation (40). Then linearizing (40) and using equality (42), we obtain the linear equation

$$
\ddot{\eta}+\beta \dot{\eta}+\mu \cos \left(\theta_{0}\right) \eta=0 .
$$

According to the Lyapunov stability theorem based on the linear approximation, solution (41) is asymptotically stable if all eigenvalues of linearized equation (43) have negative real parts. Which happens when all coefficients in (43) are positive

$$
\beta>0, \quad \mu \cos \left(\theta_{0}\right)>0
$$

due to the Routh-Hurwitz conditions. From conditions (44), assumption $\mu>0$ in (38), and equality (42), it follows for $\beta>0$ that the solutions

$$
\theta=\theta_{0}-\tau, \quad \theta_{0}=\arcsin \left(\frac{\beta}{\mu}\right)+2 \pi k
$$

are asymptotically stable, while the solutions

$$
\theta=\theta_{0}-\tau, \quad \theta_{0}=\pi-\arcsin \left(\frac{\beta}{\mu}\right)+2 \pi k
$$

are unstable, where $k$ is any integer number. For negative damping, $\beta<0$, both these solutions are unstable. From now on we will assume that the following conditions are satisfied

$$
0<\beta<\mu
$$

which ensure the existence of stable rotational solution (45) as it is seen from (42) and (44). Indeed, in order to guarantee asymptotic stability $\beta$ should be not only positive, but also strictly less than $\mu$ because of the second condition in (44), which can be transformed to inequality $\mu \cos \left(\theta_{0}\right)=\sqrt{\mu^{2}-\beta^{2}}>0$ with the use of the positive root for $\mu \cos \left(\theta_{0}\right)$ from (42). 


\subsection{Approximate rotational solutions when $\varepsilon \approx 0$ and $\omega \sim \sqrt{\varepsilon}$}

We assume that values of $\varepsilon$ and $\omega^{2}$ are small of the same order of smallness, i.e. $\varepsilon \sim \omega^{2} \ll$ 1 , so we can introduce new parameter $w=\omega^{2} / \varepsilon$. One can deduce from (38) and current assumptions that either gravity $g$ is small or the frequency of excitation $\Omega$ is high with such damping $c$ and mass $m$ so that damping coefficient $\beta \sim 1$. All small terms are in the right-hand side of equation (39). To solve equation (39) we assume that general solution of equation (39) has the form

$$
\theta=-\tau+\theta_{0}+\varepsilon \theta_{1}+\varepsilon^{2} \theta_{2}+\ldots
$$

Next the general solution is substituted into equation (39), where sines are expanded into the Taylor series with respect to $\varepsilon$. By grouping together the terms with the same powers of $\varepsilon$ and equating to zero, the set of differential equations is obtained

$$
\begin{aligned}
\ddot{\theta}_{0}+\beta \dot{\theta}_{0}+\mu \sin \left(\theta_{0}\right) & =\beta, \\
\ddot{\theta}_{1}+\beta \dot{\theta}_{1}+\mu \cos \left(\theta_{0}\right) \theta_{1} & =\sin \left(2 \tau-\theta_{0}\right)+w \sin \left(\tau-\theta_{0}-\delta\right), \\
\ddot{\theta}_{2}+\beta \dot{\theta}_{2}+\mu \cos \left(\theta_{0}\right) \theta_{2} & =\mu \sin \left(\theta_{0}\right) \theta_{1}^{2} / 2-\left(\cos \left(2 \tau-\theta_{0}\right)+w \cos \left(\tau-\theta_{0}-\delta\right)\right) \theta_{1},
\end{aligned}
$$

We have already found solution (41) for equation (49) in the previous section. Here we consider the same stable regular rotations 1:1 (with the period equal to the period of excitation) whose zero approximation is given by (45). Hence, $\theta_{0}$ is a constant. Thus, equations (50) and (51) can be written in the following way

$$
\begin{aligned}
& \ddot{\theta}_{1}+\beta \dot{\theta}_{1}+\sqrt{\mu^{2}-\beta^{2}} \theta_{1}=\sin \left(2 \tau-\theta_{0}\right)+w \sin \left(\tau-\theta_{0}-\delta\right) \\
& \ddot{\theta}_{2}+\beta \dot{\theta}_{2}+\sqrt{\mu^{2}-\beta^{2}} \theta_{2}=\beta \theta_{1}^{2} / 2-\left(\cos \left(2 \tau-\theta_{0}\right)+w \cos \left(\tau-\theta_{0}-\delta\right)\right) \theta_{1},
\end{aligned}
$$

where we denote $\mu \sin \left(\theta_{0}\right)=\beta$ and $\mu \cos \left(\theta_{0}\right)=\sqrt{\mu^{2}-\beta^{2}}$ with the use of relation (42) and the second condition in (44).

\subsubsection{First order approximation}

In consequence of conditions (47) non-homogeneous linear differential equation (52) can be presented in the following form

$$
\ddot{\theta}_{1}+\beta \dot{\theta}_{1}+\sqrt{\mu^{2}-\beta^{2}} \theta_{1}=A_{1} \cos (\tau)+B_{1} \sin (\tau)+A_{2} \cos (2 \tau)+B_{2} \sin (2 \tau)
$$

where $A_{1}=-w \cos (\delta) \beta / \mu-w \sin (\delta) \sqrt{1-\beta^{2} / \mu^{2}}, B_{1}=w \cos (\delta) \sqrt{1-\beta^{2} / \mu^{2}}-w \sin (\delta) \beta / \mu$, $A_{2}=-\beta / \mu, B_{2}=\sqrt{1-\beta^{2} / \mu^{2}}$, lower index denotes harmonics number. Equation (54) has a unique periodic solution

$$
\theta_{1}(\tau)=a_{1} \cos (\tau)+b_{1} \sin (\tau)+a_{2} \cos (2 \tau)+b_{2} \sin (2 \tau),
$$

where $\left.a_{1}=-\frac{\left(1-\sqrt{\mu^{2}-\beta^{2}}\right) A_{1}+\beta B_{1}}{\mu^{2}+1-2 \sqrt{\mu^{2}-\beta^{2}}}, b_{1}=-\frac{-\beta A_{1}+\left(1-\sqrt{\mu^{2}-\beta^{2}}\right) B_{1}}{\mu^{2}+1-2 \sqrt{\mu^{2}-\beta^{2}}}, a_{2}=-\frac{\left(4-\sqrt{\mu^{2}-\beta^{2}}\right) A_{2}+2 \beta B_{2}}{3 \beta^{2}+\mu^{2}+4\left(4-2 \sqrt{\mu^{2}-\beta^{2}}\right.}\right)$, and $b_{2}=\frac{-2 \beta A_{2}+\left(4-\sqrt{\mu^{2}-\beta^{2}}\right) B_{2}}{3 \beta^{2}+\mu^{2}+4\left(4-2 \sqrt{\mu^{2}-\beta^{2}}\right)}$. Thus, the solution for (39) in the first approximation can be 
written as follows

$$
\begin{aligned}
\theta= & -\tau+\theta_{0}-\varepsilon \frac{2 \beta \cos \left(2 \tau-\theta_{0}\right)+\left(4-\sqrt{\mu^{2}-\beta^{2}}\right) \sin \left(2 \tau-\theta_{0}\right)}{3 \beta^{2}+\mu^{2}+8\left(2-\sqrt{\mu^{2}-\beta^{2}}\right)} \\
& -\omega^{2} \frac{\beta \cos \left(\tau-\theta_{0}-\delta\right)+\left(1-\sqrt{\mu^{2}-\beta^{2}}\right) \sin \left(\tau-\theta_{0}-\delta\right)}{\mu^{2}+1-2 \sqrt{\mu^{2}-\beta^{2}}}+o(\varepsilon),
\end{aligned}
$$

where constant $\theta_{0}$ is defined in (45).

\subsubsection{Second order approximation}

Equation (54) takes the following form

$$
\ddot{\theta}_{2}+\dot{\theta}_{2}+\sqrt{\mu^{2}-\beta^{2}} \theta_{2}=\frac{A_{0}^{\prime}}{2}+\sum_{n=1}^{4}\left(A_{n}^{\prime} \cos (n \tau)+B_{n}^{\prime} \sin (n \tau)\right),
$$

where coefficients in the right-hand side are the following

$$
\begin{aligned}
& A_{0}^{\prime}=\left(b_{2}^{2}+b_{1}^{2}+a_{2}^{2}+a_{1}^{2}\right) \beta+\left(A_{1} b_{1}+A_{2} b_{2}-B_{2} a_{2}-B_{1} a_{1}\right) \\
& A_{1}^{\prime}=\left(a_{1} a_{2}+b_{1} b_{2}\right) \beta+\left(A_{1} b_{2}+A_{2} b_{1}-B_{1} a_{2}-B_{2} a_{1}\right) / 2 \\
& A_{2}^{\prime}=\left(a_{1}^{2}-b_{1}^{2}\right) \beta / 2-\left(A_{1} b_{1}+B_{1} a_{1}\right) / 2 \\
& A_{3}^{\prime}=\left(a_{1} a_{2}-b_{1} b_{2}\right) \beta-\left(A_{2} b_{1}+A_{1} b_{2}+B_{1} a_{2}+B_{2} a_{1}\right) / 2 \\
& A_{4}^{\prime}=\left(a_{2}^{2}-b_{2}^{2}\right) \beta / 2-\left(A_{2} b_{2}+B_{2} a_{2}\right) / 2 \\
& B_{1}^{\prime}=\left(a_{1} b_{2}-b_{1} a_{2}\right) \beta-\left(A_{1} a_{2}-A_{2} a_{1}+B_{1} b_{2}-B_{2} b_{1}\right) / 2 \\
& B_{2}^{\prime}=\beta a_{1} b_{1}+\left(A_{1} a_{1}-B_{1} b_{1}\right) / 2 \\
& B_{3}^{\prime}=\left(a_{1} b_{2}+b_{1} a_{2}\right) \beta+\left(A_{1} a_{2}+A_{2} a_{1}-B_{1} b_{2}-B_{2} b_{1}\right) / 2 \\
& B_{4}^{\prime}=\beta a_{2} b_{2}+\left(A_{2} a_{2}-B_{2} b_{2}\right) / 2 .
\end{aligned}
$$

Periodic solution for equation (57) has the form

$$
\begin{aligned}
\theta_{2}(\tau) & =\frac{A_{0}^{\prime}}{2 \sqrt{\mu^{2}-\beta^{2}}}-\sum_{n=1}^{4} \frac{\left(n^{2}-\sqrt{\mu^{2}-\beta^{2}}\right) A_{n}^{\prime}+n \beta B_{n}^{\prime}}{\left(n^{2}-1\right) \beta^{2}+\mu^{2}+n^{2}\left(n^{2}-2 \sqrt{\mu^{2}-\beta^{2}}\right)} \cos (n \tau) \\
& -\sum_{n=1}^{4} \frac{-n \beta A_{n}^{\prime}+\left(n^{2}-\sqrt{\mu^{2}-\beta^{2}}\right) B_{n}^{\prime}}{\left(n^{2}-1\right) \beta^{2}+\mu^{2}+n^{2}\left(n^{2}-2 \sqrt{\mu^{2}-\beta^{2}}\right)} \sin (n \tau) .
\end{aligned}
$$

Thus, second order approximate solution can be shortly written in the following form

$$
\theta=-\tau+\theta_{0}+\varepsilon \theta_{1}(\tau)+\varepsilon^{2} \theta_{2}(\tau)+o\left(\varepsilon^{2}\right),
$$

where constant $\theta_{0}$ is defined in (45), function $\theta_{1}$ in (55), and function $\theta_{2}$ in (59). In Fig. 10 it is shown how first and second order approximate solutions approach the numerical solution.

In this section the straightforward asymptotic method works since all solutions in each approximation converge to corresponding unique periodic solutions because damping $\beta$ is not small. If damping $\beta$ is small the analysis becomes more complicated since equation (49) takes the form $\ddot{\theta}_{0}+\mu \sin \left(\theta_{0}\right)=0$ and has a solution expressed in elliptic functions. One can simplify the analysis assuming that $\theta=-\tau+\varepsilon^{1 / 2} \theta_{0}+\varepsilon \theta_{1}(\tau)+\varepsilon^{3 / 2} \theta_{2}(\tau)+\ldots$ instead of $(48)$. We will use this assumption in the next section to apply the classical averaging technique to the problem of small damping $\beta \sim \sqrt{\varepsilon}$. 


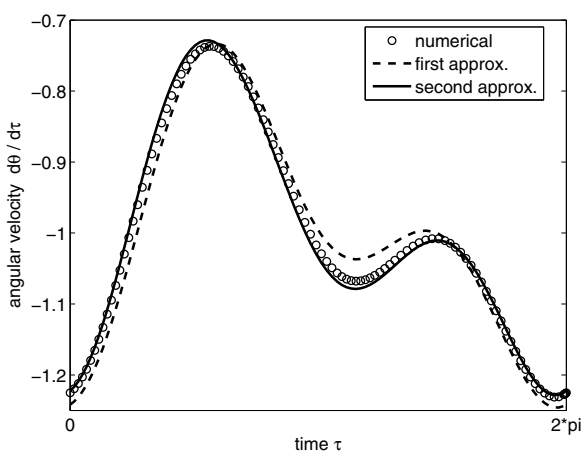

Figure 10. Angular velocities $\dot{\theta}$ calculated from the first order approximate solution (56), second order approximate solution (60), and results of numerical simulation, when damping coefficient $\beta$ is not small. Parameters: $\delta=0, \mu=1, \omega=0.3, \varepsilon=0.2, \beta=0.5$.

\subsection{Approximate rotational solutions when $\varepsilon \approx 0, \omega \sim \sqrt{\varepsilon}$, and $\beta \sim \sqrt{\varepsilon}$}

One can see in (38) that assumptions $\omega \sim \beta \sim \sqrt{\varepsilon}$ are valid for the high frequency of excitation $\Omega \sim 1 / \sqrt{\varepsilon}$ with other parameters being of order 1 . Another option is small gravity $g \sim \varepsilon$ along with small ratio $c / m \sim \sqrt{\varepsilon}$.

After change of variable $\theta=-\tau+\sqrt{\varepsilon} \vartheta$ equation (39) takes the following form

$$
\ddot{\vartheta}+\mu \vartheta-\tilde{\beta}=\mu\left(\vartheta-\frac{\sin (\sqrt{\varepsilon} \vartheta)}{\sqrt{\varepsilon}}\right)-\sqrt{\varepsilon} \tilde{\beta} \dot{\vartheta}+\sqrt{\varepsilon} \sin (2 \tau-\sqrt{\varepsilon} \vartheta)+\sqrt{\varepsilon} w \sin (\tau-\sqrt{\varepsilon} \vartheta-\delta)
$$

with small right-hand side, where we denote $\tilde{\beta}=\beta / \sqrt{\varepsilon}$ and as in the previous section $w=$ $\omega^{2} / \varepsilon$. With zero right-hand side equation (61) $\ddot{\vartheta}+\mu \vartheta-\tilde{\beta}=0$ would describe harmonic oscillations about $\tilde{\beta} / \mu$ value with frequency $\sqrt{\mu}$. After Taylor's expansion of sines in the right-hand side of (61) about $\vartheta=0$ we obtain the following equation

$$
\begin{aligned}
\ddot{\vartheta} & +(\mu+\varepsilon \cos (2 \tau)+\varepsilon w \cos (\tau-\delta)) \vartheta-\tilde{\beta} \\
& =\sqrt{\varepsilon}(\sin (2 \tau)+w \sin (\tau-\delta)-\tilde{\beta} \dot{\vartheta})+\varepsilon \mu \frac{\vartheta^{3}}{6}+o(\varepsilon),
\end{aligned}
$$

which describes oscillator with both basic and parametric excitations. To solve equation (62) we use the method of averaging $[9,18,19]$. For that purpose we write $(62)$ in the standard form of first order differential equations with small right-hand sides. First, we use Poincaré variables $q$ and $\psi$ defined via the following solution of generating system $\ddot{\vartheta}+\mu \vartheta-\tilde{\beta}=0$ which is (62) with $\varepsilon=0$

$$
\vartheta=\frac{\tilde{\beta}}{\mu}+q \cos (\psi), \quad \dot{\vartheta}=-\sqrt{\mu} q \sin (\psi) .
$$

In Poincaré variables equation (62) becomes a system of first order differential equations

$$
\dot{q}=-\frac{\sin \psi}{\sqrt{\mu}} f(\tau, q, \psi), \quad \dot{\psi}=\sqrt{\mu}-\frac{\cos \psi}{q \sqrt{\mu}} f(\tau, q, \psi),
$$


where small function $f(\tau, q, \psi)=\sqrt{\varepsilon} f_{1}(\tau, q, \psi)+\varepsilon f_{2}(\tau, q, \psi)+o(\varepsilon)$ is the right hand side of (61), where

$$
\begin{aligned}
& f_{1}(\tau, q, \psi)=\sin (2 \tau)+w \sin (\tau-\delta)+\tilde{\beta} q \sqrt{\mu} \sin (\psi), \\
& f_{2}(\tau, q, \psi)=-(\cos (2 \tau)+w \cos (\tau-\delta))\left(\frac{\tilde{\beta}}{\mu}+q \cos (\psi)\right)+\frac{\mu}{6}\left(\frac{\tilde{\beta}}{\mu}+q \cos (\psi)\right)^{3},
\end{aligned}
$$

meaning that $f(\tau, q, \psi)=O(\sqrt{\varepsilon})$. Our next assumption is that $\sqrt{\mu}-1=O(\sqrt{\varepsilon})$ which means that excitation frequency is close to the first resonant frequency of basic excitation component $\sin (\tau-\delta)$ and to the first resonant frequency of parametric excitation component $\cos (2 \tau)$ in equation (62). Thus, system (64) is transformed by $\psi=\zeta+\tau$ to the standard form

$$
\begin{aligned}
& \dot{q}=-\frac{1}{\sqrt{\mu}} \sin (\zeta+\tau) f(\tau, q, \zeta+\tau), \\
& \dot{\zeta}=\sqrt{\mu}-1-\frac{1}{q \sqrt{\mu}} \cos (\zeta+\tau) f(\tau, q, \zeta+\tau),
\end{aligned}
$$

with small right-hand side, where new slow variable $\zeta$ is often referred to as phase mismatch.

In the second approximation so called averaged equations can be obtained from the system of equations (67) and (68) as follows, see (121) in the Appendix,

$$
\begin{aligned}
\dot{Q} & =-\sqrt{\varepsilon}\left(\frac{w}{2 \sqrt{\mu}} \cos (Z+\delta)+\frac{\tilde{\beta}}{2} Q\right) \\
& +\varepsilon\left(\frac{w \tilde{\beta}}{8 \sqrt{\mu}}\left(\frac{4}{\mu}-1\right) \sin (Z+\delta)+\frac{\sin (2 Z)}{4 \sqrt{\mu}} Q\right)+o(\varepsilon) \\
\dot{Z} & =\sqrt{\mu}-1+\frac{\sqrt{\varepsilon} w}{2 \sqrt{\mu} Q} \sin (Z+\delta) \\
& +\varepsilon\left(\frac{w \tilde{\beta}}{8 \sqrt{\mu} Q}\left(\frac{4}{\mu}-1\right) \cos (Z+\delta)-\frac{\tilde{\beta}^{2}}{8}\left(\frac{2}{\mu \sqrt{\mu}}+1\right)+\frac{\cos (2 Z)}{4 \sqrt{\mu}}-\frac{\sqrt{\mu}}{16} Q^{2}\right)+o(\varepsilon),
\end{aligned}
$$

where $Q$ and $Z$ are the averaged variables corresponding to $q$ and $\zeta$.

\subsubsection{First order approximation}

Stationary solutions $(\dot{Q}=0, \dot{Z}=0)$ of (69)-(70) in the first approximation are the following

$$
Q_{\{1\}}^{2}=\frac{\omega^{2} / \varepsilon}{\mu\left(4(\sqrt{\mu}-1)^{2}+\beta^{2}\right)}, \quad Z_{\{1\}}=\arctan \left(\frac{2(\mu-1)}{\beta}\right)-\delta+2 \pi k,
$$

where we have substituted back $w=\omega^{2} / \varepsilon$ and $\tilde{\beta}=\beta / \sqrt{\varepsilon}$. Symbol arctan stands for the principal value of the function on the interval from 0 to $\pi$. Note that the phase $Z_{\{1\}}$ is determined to within $2 \pi$ rather than $\pi$, since the functions $\sin \left(Z_{\{1\}}\right)$ and $\cos \left(Z_{\{1\}}\right)$ obtained from equations (69) and (70) determine $Z_{\{1\}}$ up to an additive term $2 \pi k$. Solution of system (67)-(68) in the first approximation is $q=Q_{\{1\}}+o(1), \zeta=Z_{\{1\}}+o(1)$ so the solution of (39) is the following

$$
\theta=-\tau+\frac{\beta}{\mu}+\sqrt{\varepsilon} Q_{\{1\}} \cos \left(Z_{\{1\}}+\tau\right)+o(\sqrt{\varepsilon}),
$$


which does not contain higher harmonics observed numerically. That is why we need to proceed to the second order approximation.

\subsubsection{Second order approximation}

In the second approximation averaged equations can be obtained as described in Appendix. Stationary solutions $(\dot{Q}=0, \dot{Z}=0)$ of (69), (70) in the second approximation can be found numerically or with the absence of gravity $(\omega=0)$ analytically. Solution of system (67), (68) in the second approximation is the following, see (119),

$$
\begin{aligned}
q= & Q_{\{2\}}+\frac{\sqrt{\varepsilon}}{2 \sqrt{\mu}}\left(-\sin \left(\tau-Z_{\{2\}}\right)+\frac{w}{2} \sin \left(2 \tau+Z_{\{2\}}-\delta\right)+\frac{1}{3} \sin \left(3 \tau+Z_{\{2\}}\right)\right) \\
& +\sqrt{\varepsilon} \frac{\tilde{\beta} Q_{\{2\}}}{4} \sin \left(2 \tau+2 Z_{\{2\}}\right)+o(\sqrt{\varepsilon}), \\
\zeta= & Z_{\{2\}}+\frac{\sqrt{\varepsilon}}{2 \sqrt{\mu} Q_{\{2\}}}\left(\cos \left(\tau-Z_{\{2\}}\right)+\frac{w}{2} \cos \left(2 \tau+Z_{\{2\}}-\delta\right)+\frac{1}{3} \cos \left(3 \tau+Z_{\{2\}}\right)\right) \\
& +\sqrt{\varepsilon} \frac{\tilde{\beta}}{4} \cos \left(2 \tau+2 Z_{\{2\}}\right)+o(\sqrt{\varepsilon}) .
\end{aligned}
$$

Substitution of these expressions into (63) yields the second order approximate solution of (61), which after changes of variable $\theta=-\tau+\sqrt{\varepsilon} \vartheta$ and parameters $w=\omega^{2} / \varepsilon, \tilde{\beta}=\beta / \sqrt{\varepsilon}$ results in the approximate solution of the original equation (39)

$$
\begin{aligned}
\theta= & -\tau+\frac{\beta}{\mu}+\sqrt{\varepsilon} Q_{\{2\}} \cos \left(Z_{\{2\}}+\tau\right)+\sqrt{\varepsilon} \frac{\beta Q_{\{2\}}}{4} \sin \left(Z_{\{2\}}+\tau\right) \\
& +\frac{\omega^{2} \sin (\tau-\delta)}{4 \sqrt{\mu}}-\frac{\varepsilon \sin (2 \tau)}{3 \sqrt{\mu}}+o(\varepsilon) .
\end{aligned}
$$

Agreement of solution (75) with the numerical experiment is shown in Fig. 11. We see that the amplitude of angular velocity oscillations is much higher than that for not small $\beta$ in Fig. 10.

\section{Twirling of a hula-hoop}

A hula-hoop is a popular toy - a thin hoop that is twirled around the waist, limbs or neck. In recent decades it is widely used as an implement for fitness and gymnastic performances ${ }^{2}$ To twirl a hula-hoop the waist of a gymnast carries out a periodic motion in the horizontal plane. For the sake of simplicity we consider the two-dimensional problem disregarding the vertical motion of the hula-hoop. We assume that the waist is a circle and its center moves along an elliptic trajectory close to a circle.

Previously considered was the simple case in which a hula-hoop is treated as a pendulum with the pivot oscillating along a line, see $[37,38]$. The stationary rotations of a hula-hoop

\footnotetext{
${ }^{2}$ The same model lies in the basis of some industrial machinery such as vibrating cone crushers designed for crushing hard brittle materials, see [33].
} 


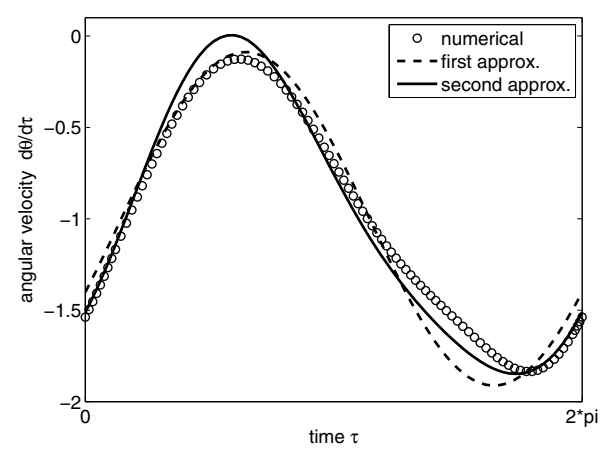

Figure 11. Angular velocity $\dot{\theta}$ calculated from the first order approximate solution (72) and second order approximate solution (75) compared with the results of numerical simulations in the case of small damping $\beta$. Parameters: $\delta=0, \mu=1, \omega=0.3, \varepsilon=0.2, \beta=0.01$. Steady state averaged variables $Q$ and $\mathrm{Z}$ are given by expressions in (71) for the first approximation while for the second approximation they are obtained numerically $(Q=2.0348, Z=2.6838)$ from the second order averaged equations (69), (70).

excited in two directions have been studied by an approximate method of separate motions in [33]. The similar problem of the spinner mounted loosely on a pivot with a prescribed bi-directional motion has been treated numerically and experimentally in [39].

Here we derive the exact solutions in the case of a circular trajectory of the waist center and approximate solutions in the case of an elliptic trajectory. We also check the condition of keeping contact with the waist during twirling.
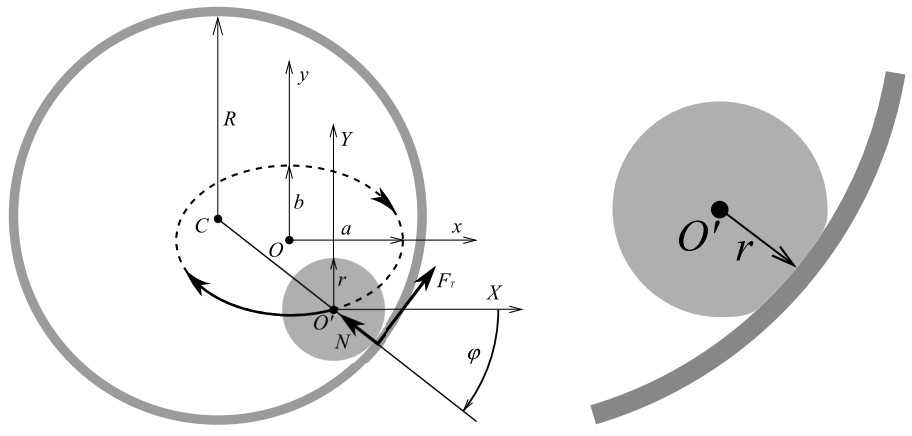

Figure 12. A hula-hoop with the radius $R$ twirling with the angle $\varphi$ around a circular waist (shaded) with the radius $r$. The center $O^{\prime}$ of the waist moves along the elliptic curve $x=a \sin \omega t, y=b \cos \omega t$ with the fixed center $O$. The hula-hoop acts on the waist with normal force $N$ and tangential friction force $F_{T}$. There is also a rolling resistance due to the waist deformation (right).

\subsection{Main relations}

We assume that the center $O^{\prime}$ of a gymnast's waist moves in time according to the elliptic law $x=a \sin \omega t, y=b \cos \omega t$ with the amplitudes $a, b$ and the excitation frequency $\omega>0$, Fig. 12 . 
The equations of motion in the waist-fixed coordinate system take the following form

$$
\begin{aligned}
I_{C} \ddot{\theta}+k \dot{\theta} & =-F_{T} R-d N \operatorname{sign}(\dot{\theta}), \\
m(R-r) \ddot{\varphi} & =m(\ddot{x} \sin \varphi+\ddot{y} \cos \varphi)+F_{T}, \\
m(R-r) \dot{\varphi}^{2} & =N+m(\ddot{x} \cos \varphi-\ddot{y} \sin \varphi),
\end{aligned}
$$

where $\theta$ is the rotation angle around center of mass $C, I_{C}=m R^{2}$ is the central moment of inertia of the hula-hoop, $\varphi$ is the angle between axis $x$ and radius $C O^{\prime}, r$ is the radius of the waist, $m$ and $R$ are the mass and radius of the hula-hoop. Equation (76) describes change of angular momentum due to linear viscous damping with coefficient $k$, rolling drag (rolling resistance) with coefficient $d$, and the tangential friction force $F_{T}$ between the waist and the hoop. Equations (77) and (78) describe the motion of the hula-hoop in the longitudinal and transverse directions to the radius $\mathrm{CO}^{\prime}$, where $N$ is the normal reaction force of the hula-hoop to the waist. Equations (77) and (78) contain additional inertial forces since the waist-fixed reference system is noninertial.

Assuming that slipping at the point of contact is absent we obtain the kinematic relation

$$
(R-r) \dot{\varphi}=R \dot{\theta} .
$$

We exclude from equations (76) and (77) the force $F_{T}$ and with relation (79) obtain the equation of motion

$$
\begin{aligned}
\ddot{\varphi} & +\frac{k}{2 m R^{2}} \dot{\varphi}+\frac{d}{2 R} \operatorname{sign} \dot{\varphi}\left(\dot{\varphi}^{2}+\frac{\omega^{2}(a \sin \omega t \cos \varphi-b \cos \omega t \sin \varphi)}{R-r}\right) \\
& +\frac{\omega^{2}(a \sin \omega t \sin \varphi+b \cos \omega t \cos \varphi)}{2(R-r)}=0 .
\end{aligned}
$$

From equation (78) we find the normal force and imply the condition $N>0$ as

$$
(R-r) \dot{\varphi}^{2}+\omega^{2}(a \sin \omega t \cos \varphi-b \cos \omega t \sin \varphi)>0
$$

which means that the hula-hoop during its motion keeps contact with the waist of the gymnast.

We introduce new time $\tau=\omega t$ and non-dimensional parameters

$$
\gamma=\frac{k}{2 m R^{2} \omega}, \quad \delta=\frac{d}{2 R}, \quad \varepsilon=\frac{a-b}{4(R-r)}, \quad \mu=\frac{a+b}{4(R-r)},
$$

where $\gamma$ and $\delta$ are the damping and rolling resistance coefficients, $\mu$ and $\varepsilon$ are the excitation parameters. Relation between $\mu$ and $\varepsilon$ determines the form of ellipse - the trajectory of the waist center. For $\varepsilon=\mu$ the trajectory is a line, and for $\varepsilon=0$ it is a circle. Then equation (80) and inequality (81) take the form

$$
\begin{aligned}
\ddot{\varphi} & +\gamma \dot{\varphi}+\delta \dot{\varphi}|\dot{\varphi}|+\mu \cos (\varphi-\tau)-2 \mu \delta \operatorname{sign}(\dot{\varphi}) \sin (\varphi-\tau) \\
& =\varepsilon \cos (\varphi+\tau)-2 \varepsilon \delta \operatorname{sign}(\dot{\varphi}) \sin (\varphi+\tau), \\
\dot{\varphi}^{2} & -2 \mu \sin (\varphi-\tau)+2 \varepsilon \sin (\varphi+\tau)>0
\end{aligned}
$$

where the dot means differentiation with respect to the time $\tau$. 


\subsection{Exact solutions}

When the waist center moves along a circle $(a=b$, i.e. $\varepsilon=0)$ equation (83) takes the following form

$$
\ddot{\varphi}+\gamma \dot{\varphi}+\delta \dot{\varphi}|\dot{\varphi}|+\mu \cos (\varphi-\tau)-2 \mu \delta \operatorname{sign}(\dot{\varphi}) \sin (\varphi-\tau)=0
$$

and has the exact solution [6]

$$
\varphi=\tau+\varphi_{0}
$$

with the constant initial phase $\varphi_{0}$ given by the equation

$$
\gamma+\delta+\mu \cos \varphi_{0}-2 \mu \delta \sin \varphi_{0}=0 .
$$

Therefore, solution (86) exists only under the condition $|\gamma+\delta| \leq|\mu| \sqrt{1+4 \delta^{2}}$, so we find from equation (87)

$$
\varphi_{0}+\arccos \left(\frac{1}{\sqrt{1+4 \delta^{2}}}\right)= \pm \arccos \left(-\frac{\gamma+\delta}{\mu \sqrt{1+4 \delta^{2}}}\right)+2 \pi n, \quad n=0,1,2, \ldots
$$

provided that $\mu \neq 0$. Solutions (86), (88) correspond to the rotation of the hula-hoop with the constant angular velocity equal to the excitation frequency $\omega$.

\subsubsection{Stability of the exact solutions}

Let us investigate the stability of the obtained solutions. For this purpose we take the angle $\varphi$ in the form $\varphi=\tau+\varphi_{0}+\eta(\tau)$ where $\eta(\tau)$ is a small quantity, and substitute it into equation (85). Taking linearization with respect to $\eta$ and with the use of (87) we obtain a linear equation

$$
\ddot{\eta}+(\gamma+2 \delta) \dot{\eta}-\mu\left(\sin \varphi_{0}+2 \delta \cos \varphi_{0}\right) \eta=0 .
$$

According to Lyapunov's theorem on the stability based on a linear approximation [17] solution (86), (88) is asymptotically stable if all the eigenvalues of linearized equation (89) have negative real parts. From Routh-Hurwitz criterion [17] we obtain the stability conditions as

$$
\gamma+2 \delta>0, \quad \mu\left(\sin \varphi_{0}+2 \delta \cos \varphi_{0}\right)<0 .
$$

Without loss of generality we assume $\mu>0$ since the case $\mu<0$ can be reduced to the previous one by the time transformation $\tau^{\prime}=\tau+\pi$ in equation (83). The second condition in (90) can be written as $\sin \left(\varphi_{0}+\arccos \left(1 / \sqrt{1+4 \delta^{2}}\right)\right)<0$. Thus, from conditions (90), relation

(88) and due to the assumption $\mu>0$ we find that for $0<\gamma+2 \delta<\delta+\mu \sqrt{1+4 \delta^{2}}$ solution (86) with

$$
\varphi_{0}=-\arccos \left(-\frac{\gamma+\delta}{\mu \sqrt{1+4 \delta^{2}}}\right)-\arccos \left(\frac{1}{\sqrt{1+4 \delta^{2}}}\right)+2 \pi n, \quad n=0,1,2, \ldots
$$

is asymptotically stable, and solution (86) with

$$
\varphi_{0}=\arccos \left(-\frac{\gamma+\delta}{\mu \sqrt{1+4 \delta^{2}}}\right)-\arccos \left(\frac{1}{\sqrt{1+4 \delta^{2}}}\right)+2 \pi n, \quad n=0,1,2, \ldots
$$

is unstable. 


\subsubsection{Condition for hula-hoop's contact with the waist}

Let us verify for the exact solutions (86), (88) the condition of twirling without losing contact (84) which takes the form

$$
\mu \sin \varphi_{0}<\frac{1}{2}
$$

The second stability condition in (90) can be rewritten with the use of (87) as follows

$$
\mu \sin \varphi_{0}<\frac{2 \delta(\gamma+\delta)}{1+4 \delta^{2}}
$$

Thus, stable solution (86), (91) provides asymptotically stable twirling of the hula-hoop with the constant angular velocity $\omega$ without losing contact with the waist of the gymnast under the condition $\gamma \delta<1 / 4$.

Without rolling resistance $\delta=0$ condition (93) is always satisfied for the stable solution (86), (91). While for unstable solution (86), (92) we have $\mu \sin \varphi_{0}=\sqrt{\mu^{2}-\gamma^{2}}$ so condition (93) holds only if $\mu<\sqrt{1 / 4+\gamma^{2}}$. The phase $\varphi_{0}$ of the stable solution belongs to the interval $[-\pi,-\pi / 2] \bmod 2 \pi$, and for vanishing damping $\gamma \rightarrow+0$ the phase tends to $-\pi / 2$. Below we will show that this phase inequality also holds for the approximate solutions. This is how to twirl a hula-hoop!

\subsection{Approximate solutions}

Let us find approximate solutions for the case of close but not equal amplitudes $a \approx b$. For the sake of simplicity from now on we will keep $\delta=0$ and assume that $a \geq|b|$ which means $\varepsilon \geq 0, \mu \geq 0$. Taking $\varepsilon$ as a small parameter we apply perturbation method assuming that the exact solution $\varphi_{S}(\tau)$ of (83) can be expressed in a series

$$
\varphi_{s}(\tau)=\tau+\varphi_{0}+\varepsilon \varphi_{1}(\tau)+o(\varepsilon) .
$$

After substitution of series (94) in (83) and grouping the terms by equal powers of $\varepsilon$ we derive the following chain of equations

$$
\begin{array}{ll}
\varepsilon^{0}: & \gamma+\mu \cos \left(\varphi_{0}\right)=0 \\
\varepsilon^{1}: & \ddot{\varphi}_{1}+\gamma \dot{\varphi}_{1}-\mu \sin \left(\varphi_{0}\right) \varphi_{1}=\cos \left(\varphi_{0}+2 \tau\right)
\end{array}
$$

Taking solution of equation (95) for $\mu>0$

$$
\varphi_{0}=-\arccos (-\gamma / \mu)+2 \pi n, \quad n=1,2, \ldots
$$

corresponding to the stable unperturbed solution (86), (91) we write equation (96) as

$$
\ddot{\varphi}_{1}+\gamma \dot{\varphi}_{1}+\sqrt{\mu^{2}-\gamma^{2}} \varphi_{1}=\cos \left(\varphi_{0}+2 \tau\right) .
$$

It has a unique periodic solution

$$
\varphi_{1}(\tau)=C \sin \left(2 \tau+\varphi_{0}\right)+D \cos \left(2 \tau+\varphi_{0}\right),
$$

where constants $C$ and $D$ are defined as follows

$$
C=\frac{2 \gamma}{\mu^{2}+3 \gamma^{2}-8 \sqrt{\mu^{2}-\gamma^{2}}+16}, D=\frac{-4+\sqrt{\mu^{2}-\gamma^{2}}}{\mu^{2}+3 \gamma^{2}-8 \sqrt{\mu^{2}-\gamma^{2}}+16} .
$$




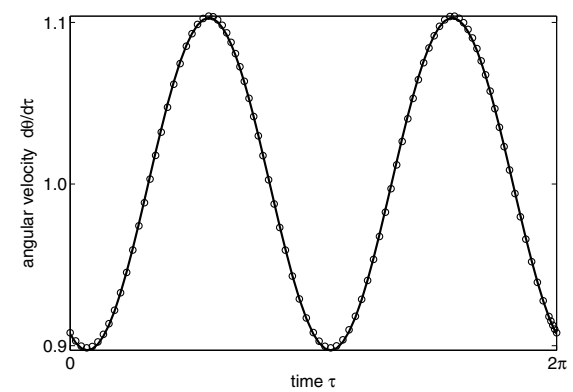

Figure 13. Comparison between the approximate analytical (solid line) and numerical (circles) results for parameters: $\mu=1.2, \varepsilon=0.2$, and $\gamma=1$. Initial conditions for numerical calculations are $\theta(0)=-2.5389, \dot{\theta}(0)=0.90802$.

We see that the approximate solution $\varphi(\tau)=\tau+\varphi_{0}+\varepsilon \varphi_{1}(\tau)$ with (97), (99), (100) differs from the exact solution (86), (91) of the unperturbed system by small vibrating terms of frequency 2 , see (99). Note that the approximate solutions were obtained with the assumption that the excitation amplitudes and damping are not small.

\subsubsection{Stability of the approximate solutions}

To find the stability conditions for solution (94), (97), (99) we take a small variation to the solution of (94) $\varphi=\varphi_{s}+u$ and substitute this expression into (83). After linearization with respect to $u$ and keeping only terms of first order we obtain a linear equation

$$
\ddot{u}+\gamma \dot{u}+\left(-\mu\left(\sin \varphi_{0}+\varepsilon \varphi_{1} \cos \varphi_{0}\right)+\varepsilon \sin \left(2 \tau+\varphi_{0}\right)\right) u=0,
$$

where $\varphi_{0}$ is given by expression (97). Equation (101) can be written in the form of damped Mathieu-Hill equation as

$$
\ddot{u}+\gamma \dot{u}+(p+\varepsilon \Phi(2 \tau)) u=0,
$$

where $p=-\mu \sin \varphi_{0}=\sqrt{\mu^{2}-\gamma^{2}}, \Phi(2 \tau)=(\gamma C+1) \sin \left(2 \tau+\varphi_{0}\right)+\gamma D \cos \left(2 \tau+\varphi_{0}\right)$. Then the stability condition (absence of parametric resonance at all frequencies $\sqrt{p}$ ) is given by the inequalities [17]

$$
\varepsilon<\frac{2 \gamma}{\sqrt{(\gamma C+1)^{2}+\gamma^{2} D^{2}}}
$$

with $C$ and $D$ defined in (100). This is the inequality to the problem parameters $\gamma, \varepsilon$ and $\mu$.

\subsubsection{Condition for hula-hoop's contact with the waist}

The condition of twirling without losing contact (84) takes the following form

$$
\varepsilon<\frac{1+2 \sqrt{\mu^{2}-\gamma^{2}}}{2} \sqrt{\frac{\mu^{2}+3 \gamma^{2}-8 \sqrt{\mu^{2}-\gamma^{2}}+16}{\mu^{2}+8 \gamma^{2}-12 \sqrt{\mu^{2}-\gamma^{2}}+36}} .
$$

Conditions (103), (104) imply restrictions to $\varepsilon$, i.e. how much the elliptic trajectory of the waist center differs from the circle. 


\subsubsection{Comparison with numerical simulations}

In Fig. 13 the approximate analytical solution is presented and compared with the results of numerical simulation for the case when the excitation parameter $\mu$ and damping coefficient $\gamma$ are not small.

\subsection{Small excitation amplitudes and damping}

It is interesting to consider the case when the excitation amplitudes and damping coefficient are small having the same order as $\varepsilon$. Then we introduce new parameters $\tilde{\mu}=\mu / \varepsilon$ and $\tilde{\gamma}=$ $\gamma / \varepsilon$ and assume that the solution has the form

$$
\varphi(\tau)=\rho \tau+\varphi_{0}(\tau)+\varepsilon \varphi_{1}(\tau)+o(\varepsilon),
$$

where $\rho$ is the angular velocity of rotation, and the functions $\varphi_{0}(\tau), \varphi_{1}(\tau)$ are supposed to be bounded.

We substitute expression (105) into (83) and equating terms of the same powers of $\varepsilon$ obtain the following equations

$$
\begin{aligned}
& \varepsilon^{0}: \ddot{\varphi}_{0}=0 \\
& \varepsilon^{1}: \ddot{\varphi}_{1}=\cos \left(\varphi_{0}+\tau+\rho \tau\right)-\tilde{\mu} \cos \left(\varphi_{0}-\tau+\rho \tau\right)-\tilde{\gamma} \dot{\varphi}_{0}-\tilde{\gamma} \rho .
\end{aligned}
$$

From equation (106) we get that function $\varphi_{0}(\tau)$ can remain bounded only if it is constant $\left(\varphi_{0}(\tau) \equiv \varphi_{0}=\right.$ const). Then equation (107) can have bounded solutions $\varphi_{1}(\tau)$ only when $\rho$ takes the values $-1,0,1$. Thus, besides clockwise rotation we have also counterclockwise rotation $\rho=-1$, and no rotational solution $\rho=0$. The letter is not interesting, so we omit it.

\subsubsection{Clockwise rotation}

For clockwise rotation $\rho=1$, see Fig. 14 a), from equations (105), (106), and (107) we obtain in the first approximation the solution

$$
\begin{gathered}
\varphi_{*}(\tau)=\tau+\varphi_{0}+\varepsilon \varphi_{1}(\tau), \\
\varphi_{1}(\tau)=-\frac{1}{4} \cos \left(\varphi_{0}+2 \tau\right), \quad \cos \varphi_{0}=-\frac{\gamma}{\mu},
\end{gathered}
$$

where function $\varphi_{1}(\tau)$ is found up to the addition of a constant, which we have set to zero for determinacy. Thus, we let only $\varphi_{0}$ contain a constant term of the solution. The first expression in (109) is the special case of (99), (100) when $\mu=\gamma=0$.

To verify the stability conditions for solution (108) we use damped Mathieu-Hill equation (102) and for the case of small damping and excitation amplitudes get $\gamma>0, \sin \varphi_{0}<0$, see [17]. These conditions are similar with inequalities (90) derived for the undisturbed exact solution. Thus, the stable solution (108) with $\varphi_{0}=-\arccos (-\gamma / \mu)+2 \pi n$ exists for

$$
0<\gamma<\mu \text {. }
$$

For solution (108) condition (84) of keeping contact in the first approximation reads

$$
\varepsilon<\frac{1+2 \sqrt{\mu^{2}-\gamma^{2}}}{3}
$$

and holds true for sufficiently small $\varepsilon$. 

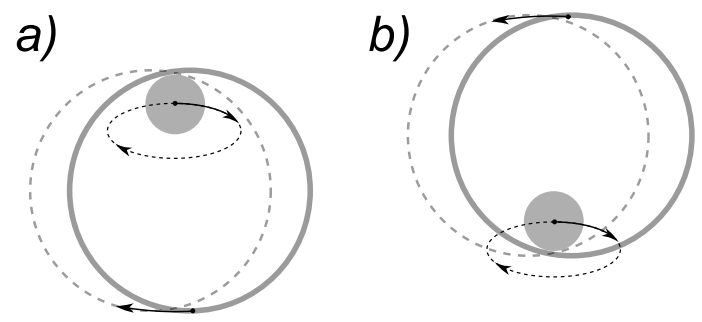

Figure 14. Stable twirling of the hula-hoop for the cases: a) direct twirling b) inverse twirling.

\subsubsection{Counterclockwise rotation}

For counterclockwise rotation $\rho=-1$ in Fig. 14 b) we obtain in the first approximation the solution

$$
\varphi_{*}(\tau)=-\tau+\varphi_{0}+\frac{\mu}{4} \cos \left(\varphi_{0}-2 \tau\right), \quad \cos \varphi_{0}=-\frac{\gamma}{\varepsilon},
$$

with the stability conditions $\gamma>0, \sin \varphi_{0}>0$. Thus, the stable counterclockwise rotation (112) with $\varphi_{0}=\arccos (-\gamma / \varepsilon)+2 \pi n$ exists for

$$
0<\gamma<\varepsilon
$$

For this case condition (84) takes the form similar to (111) and holds true for sufficiently small $\mu$

$$
\mu<\frac{1+2 \sqrt{\varepsilon^{2}-\gamma^{2}}}{3}
$$

\subsubsection{Coexistence of clockwise and counterclockwise rotations}

It follows from conditions (110), (113) that stable clockwise and counterclockwise rotations (108), (109) and (112) coexist if the following conditions are satisfied

$$
0<\gamma<\min \{\varepsilon, \mu\} \text {. }
$$

Conditions (115) in physical variables take the form

$$
0<2 k \frac{R-r}{R^{2} \omega m}<a-|b|,
$$

meaning that the trajectory of the waist should be sufficiently prolate. Coexisting clockwise and counterclockwise rotations are illustrated in Fig. 14.

\subsubsection{Comparison with numerical simulations}

In Fig. 15 the approximate analytical solutions for rotations in both directions are presented and compared with the results of numerical simulation for the case of small excitation parameters $\mu, \varepsilon$ and the damping coefficient $\gamma$. The values of $\mu, \varepsilon$ correspond to the dimensional parameters $a=15 \mathrm{~cm}, b=10 \mathrm{~cm}, r=10 \mathrm{~cm}, R=50 \mathrm{~cm}$. 

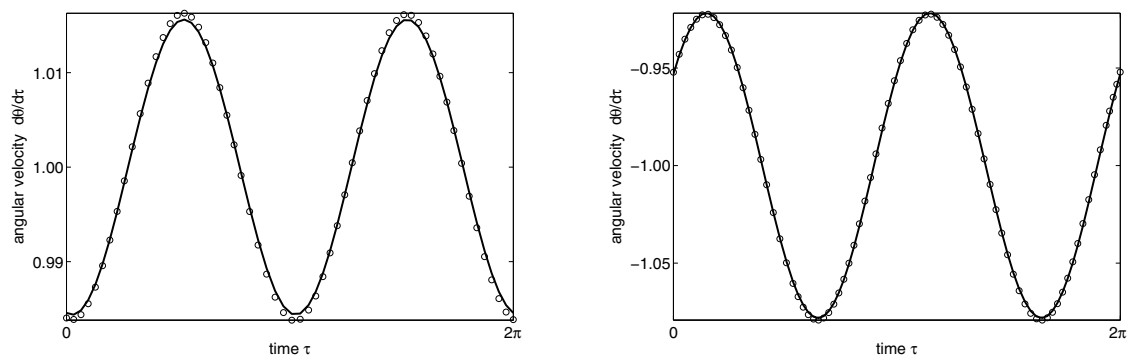

Figure 15. Comparison between the approximate analytical (solid line) and numerical (circles) results for small excitation amplitudes and damping coefficient for clockwise (left) and counterclockwise (right) rotations, for parameters: $\mu=5 / 32, \varepsilon=1 / 32$, and $\gamma=1 / 40$. Initial conditions for numerical calculations are $\theta(0)=-1.7301, \dot{\theta}(0)=0.98403$ (left) and $\theta(0)=2.4696, \dot{\theta}(0)=-0.95212$ (right).

\section{Conclusions}

In section 2 we showed that the pendulum with periodically varying length exhibits diversity of behavior types. We recognized that the analytical stability boundaries of the vertical position of the pendulum and the frequency-response curve for limit cycles are in a good agreement with the numerical results. The second resonance zone appeared to be empty. The stability conditions of limit cycles are derived based on direct use of Lyapunov's theorem on stability of periodic solutions. We found numerically regular rotation, oscillation, and rotation-oscillation regimes with various periods and mean angular velocities of the pendulum including high-speed rotations and rotations with fractional relative velocities (it is rotation-oscillation regime when the pendulum makes regular sequence of rotations in both directions). We derived analytically the conditions for existence of regular rotation and oscillation regimes which agree with the numerical results. Domains for chaotic motions are found and analyzed numerically in the parameter space via calculation of Lyapunov exponents and bifurcation diagrams. Basins of attractions of different regimes of the pendulum motion were plotted and analyzed.

In section 3 we studied the planar rotational motion of the pendulum with the pivot oscillating both vertically and horizontally when the trajectory of the pivot is an ellipse close to a circle. The analysis of motion was based on the exact rotational solutions in the case of circular pivot trajectory and zero gravity. The conditions for existence and stability of such solutions were derived. Assuming that the amplitudes of excitations are not small while the pivot trajectory has small ellipticity the approximate solutions were found both for large and small linear damping. Comparison between approximate and numerical solutions was made for different values of the damping parameter demonstrating good accuracy of the method involved.

Finally, in section 4 we assumed that the waist of a sportsman twirling a hula hoop is a circle and its center moves along an elliptic trajectory close to a circle. We studied the system with both small and not small linear viscous damping as well as with some rolling resistance. For the case of the circular trajectory, two families of the exact solutions were obtained, similar to those in section 3. Both of them correspond to twirling of the hula-hoop with a 
constant angular speed equal to the speed of the excitation. We showed that one family of the solutions is stable, while the other one is unstable. These exact solutions allowed us to obtain the approximate solutions for the case of an elliptic trajectory of the waist. An interesting effect of inverse twirling was described when the waist moves in opposite direction to the hula-hoop rotation. It is shown that the approximate analytical solutions agree with the results of numerical simulation.

\section{Acknowledgements}

The authors express their gratitude to Angelo Luongo for his contribution to dynamics of a pendulum with variable length and fruitful discussions on nonlinear mechanics.

\section{Author details}

Belyakov Anton

Institute of Mechanics, Lomonosov Moscow State University (MSU), Moscow, Russia

Institute of Mathematical Methods in Economics, Vienna University of Technology, Vienna, Austria

Seyranian Alexander P.

Institute of Mechanics, Lomonosov Moscow State University (MSU), Moscow, Russia

\section{Appendix. The standard method of averaging}

The standard averaging method is a straightforward procedure and in the literature $[9,18]$ usually only two first approximations are written down. We use the averaging method down to the fifth approximation and feel obliged to present the resulting formulas.

The standard averaging method finds the transformation of the equation system with small and $2 \pi$ time periodic right hand side

$$
\dot{x}=f_{1}(x, t)+f_{2}(x, t)+\ldots+f_{6}(x, t)
$$

into autonomous system (also called averaged system) which could be solved analytically

$$
\dot{X}=F_{1}(X)+F_{2}(X)+\ldots+F_{6}(X)+\ldots,
$$

where the lower index denotes the order of smallness with respect to one. A new variable $X$ is presented in the following series

$$
x=X+u_{1}(X, t)+u_{2}(X, t)+\ldots+u_{5}(X, t)+\ldots,
$$

which is the approximate solution of system (117) and, hence, of the transformed system (118). The functions $u_{i}$ and $F_{i}$ can be found one by one after differentiating (119) with respect to time, substituting there expressions (117) and (118), expanding the functions $f_{i}$ around $X$ into Taylor's series, and collecting there terms of the same order. 
Averaging operator is defined as follows

$$
\langle\cdot\rangle=\left.\lim _{T \rightarrow \infty} \frac{1}{T} \int_{0}^{T} \cdot\right|_{x=X} d \tau=\left.\frac{1}{2 \pi} \int_{0}^{2 \pi} \cdot\right|_{x=X} d \tau .
$$

We also define integral operator $\{\cdot\}$ with the following expression

$$
\{f(x, \tau)\}=\int(f(x, \tau)-\langle f(x, \tau)\rangle) d \tau,
$$

which is such an antiderivative that satisfies the condition $\langle\{f(x, \tau)\}\rangle=\{\langle f(x, \tau)\rangle\}=0$. Latter condition is necessary to obviate an ambiguity. We define following vector product operators

$$
f u_{k}=\sum_{i=1}^{n} \frac{\partial f}{\partial x_{i}} u_{k}^{i}, \quad f u_{k, m}=\sum_{i, j=1}^{n} \frac{\partial^{2} f}{\partial x_{i} \partial x_{j}} u_{k}^{i} u_{m}^{j}, \ldots
$$

and so on, where $i$ and $j$ are the indices of vector components placed in $u_{k}^{i}$ and $u_{m}^{j}$ on the top not to confuse it with smallness order indices $k$ and $m ; n$ is the length of vectors $f, u_{k}$ and $u_{m}$. Hence, we can write recurrent expressions

$$
\begin{aligned}
& F_{1}(X)=\left\langle f_{1}(x, t)\right\rangle, \\
& u_{1}(X, t)=\left\{f_{1}(x, t)\right\}+U_{1}(X), \quad F_{2}(X)=\left\langle f_{2}(x, t)+f_{1}(x, t) u_{1}(X, t)\right\rangle, \\
& u_{2}=\left\{f_{2}+f_{1} u_{1}-u_{1} F_{1}\right\}+U_{2}, \quad F_{3}=\left\langle f_{3}+f_{1} u_{2}+f_{2} u_{1}+\frac{1}{2} f_{1} u_{1,1}\right\rangle, \\
& u_{3}=\left\{f_{3}+f_{1} u_{2}+f_{2} u_{1}+\frac{1}{2} f_{1} u_{1,1}-u_{1} F_{2}-u_{2} F_{1}\right\}+U_{3}, \\
& F_{4}=\left\langle f_{4}+f_{1} u_{3}+f_{2} u_{2}+f_{3} u_{1}+f_{1} u_{1,2}+\frac{1}{2} f_{2} u_{1,1}+\frac{1}{6} f_{1} u_{1,1,1}\right\rangle, \\
& u_{4}=\left\{f_{4}+f_{1} u_{3}+f_{2} u_{2}+f_{3} u_{1}+f_{1} u_{1,2}+\frac{1}{2} f_{2} u_{1,1}+\frac{1}{6} f_{1} u_{1,1,1}\right. \\
&\left.-u_{1} F_{3}-u_{2} F_{2}-u_{3} F_{1}\right\}+U_{4}, \\
& F_{5}=\left\langle f_{5}+f_{1} u_{4}+f_{2} u_{3}+f_{3} u_{2}+f_{4} u_{1}+f_{1} u_{1,3}+f_{2} u_{1,2}\right. \\
&\left.+\frac{1}{2} f_{1} u_{2,2}+\frac{1}{2} f_{3} u_{1,1}+\frac{1}{2} f_{1} u_{1,1,2}+\frac{1}{6} f_{2} u_{1,1,1}+\frac{1}{24} f_{1} u_{1,1,1,1}\right\rangle, \\
& u_{5}=\left\{f_{5}+f_{1} u_{4}+f_{2} u_{3}+f_{3} u_{2}+f_{4} u_{1}+f_{1} u_{1,3}+f_{2} u_{1,2}\right. \\
&+\frac{1}{2} f_{1} u_{2,2}+\frac{1}{2} f_{3} u_{1,1}+\frac{1}{2} f_{1} u_{1,1,2}+\frac{1}{6} f_{2} u_{1,1,1}+\frac{1}{24} f_{1} u_{1,1,1,1} \\
&\left.-u_{1} F_{4}-u_{2} F_{3}-u_{3} F_{2}-u_{4} F_{1}\right\}+U_{5},
\end{aligned}
$$




$$
\begin{aligned}
F_{6}= & \left\langle f_{6}+f_{1} u_{5}+f_{2} u_{4}+f_{3} u_{3}+f_{4} u_{2}+f_{5} u_{1}+f_{1} u_{1,4}+f_{1} u_{2,3}+f_{2} u_{1,3}+f_{3} u_{1,2}\right. \\
& +\frac{1}{2} f_{2} u_{2,2}+\frac{1}{2} f_{4} u_{1,1}+\frac{1}{2} f_{1} u_{1,2,2}+\frac{1}{2} f_{2} u_{1,1,2}+\frac{1}{6} f_{3} u_{1,1,1}+\frac{1}{6} f_{1} u_{1,1,1,2} \\
& \left.+\frac{1}{24} f_{2} u_{1,1,1,1}+\frac{1}{120} f_{1} u_{1,1,1,1,1}\right\rangle,
\end{aligned}
$$

where we similarly denote $u_{k} F_{m}=\sum_{i=1}^{n} \frac{\partial u_{k}}{\partial x_{i}} F_{m}^{i}$. Functions $U_{k}=U_{k}(X)$ can be chosen arbitrarily, so for convenience we set $U_{k}(X) \equiv 0$. Thus, knowing the functions $F_{i}$ from expressions (120)-(128) we can write averaged system (118), which is simpler than the original system (117). If we solve averaged system (118) we are able to write the approximate solution (119) of system (117) substituting slow variables $X(t)$ into the functions $u_{i}(X, t)$ obtained from (121)-(127). Since the functions $u_{i}(X, t)$ are periodic with respect to time $t$ the behavior of slow variables determines the behavior of the approximate solution. It means that we can study stability of the approximate solutions by stability of the regular solutions of averaged system (118).

\section{References}

[1] Seyranian A P (2004) The Swing: Parametric Resonance. Journal of applied mathematics and mechanics 68: 757-764.

[2] Seyranian A P, Belyakov A O (2008) Swing Dynamics. Doklady physics 53(7): 388-394.

[3] Belyakov A O, Seyranian A P, Luongo A (2009) Dynamics of the Pendulum with Periodically Varying Length. Physica D 238: 1589-1597.

[4] Belyakov A O, Seyranian A P (2010) On Nonlinear Dynamics of the Pendulum with Periodically Varying Length. Mechanics of machines, BulKToMM 18(87): 21-28.

[5] Belyakov A O (2011) On Rotational Solutions for Elliptically Excited Pendulum. Physics letters A 375: 2524-2530.

[6] Belyakov A O, Seyranian A P (2010) The Hula-Hoop Problem. Doklady physics 55: 99-104.

[7] Seyranian A P, Belyakov A O (2011) How to Twirl a Hula Hoop. American j. physics 79: 712-715.

[8] Kauderer H (1958) Nichtlineare Mechanik. Berlin: Springer.

[9] Bogolyubov N N, Mitropolsky Yu A (1961) Asymptotic Methods in the Theory of Non-Linear Oscillations. New York: Gordon and Breach.

[10] Panovko Ya G, Gubanova I I. (1987) Stability and Oscillations of Elastic Systems. Modern Concepts, Paradoxes and Mistakes. Moscow: Nauka.

[11] Magnus K (1976) Schwingungen. Eine Einfuhrung in die theoretische Behandlung von Schwingungensproblemen. Stuttgart: J.Teubner.

[12] Bolotin V V (1999) Vibrations in Engineering. A Handbook, Vol. 1. Oscillations of Linear Systems. Moscow: Mashinostroenie.

[13] Pinsky M F, Zevin A A (1999) Oscillations of a Pendulum with a Periodically Varying Length and a Model of Swing. International journal of non-linear mechanics 34: 105-109. 
[14] Zevin A A, Filonenko L A (2007) Qualitative Study of Oscillations of a Pendulum with Periodically Varying Length and a Mathematical Model of Swing. Journal of applied mathematics and mechanics 71: 989-1003.

[15] Bozduganova V S, Vitliemov V G (2009) Dynamics of Pendulum with Variable Length and Dry Friction as a Simulator of a Swing. Mechanics of machines 17(82): 45-48.

[16] Seyranian A P (2001) Resonance Domains for the Hill Equation with Allowance for Damping. Doklady physics 46: 41-44.

[17] Seyranian A P, Mailybaev A A (2003) Multiparameter Stability Theory with Mechanical Applications. New Jersey: World Scientific.

[18] Volosov V M, Morgunov B I (1971) Averaging Method in the Theory of Nonlinear Oscillatoratory Systems. Moscow: MSU.

[19] Thomsen J J (2003) Vibrations and Stability. Advanced Theory, Analysis and Tools. Berlin: Springer.

[20] Awrejcewicz J, Krysko V A (2006) Introduction to Asymptotic Methods. Boca Raton, New York: Chapman and Hall, CRC Press.

[21] Nusse H E, Yorke A Y (1997) Dynamics: Numerical Explorations. New York: Springer.

[22] Szemplinska-Stupnicka W, Tyrkiel E, Zubrzycki A (2000) The Global Bifurcations that Lead to Transient Tumbling Chaos in a Parametrically Driven Pendulum. International journal of bifurcation and chaos 10: 2161-2175.

[23] Xu Xu, Wiercigroch M, Cartmell M P (2005) Rotating Orbits of a Parametrically-Excited Pendulum. Chaos solitons \& fractals 23: 1537-1548.

[24] Lenci S, Pavlovskaia E, Rega G, Wiercigroch M (2008) Rotating Solutions and Stability of Parametric Pendulum by Perturbation Method. Journal of sound and vibration 310: 243-259.

[25] Awrejcewicz J, Kudra G, Wasilewski G (2007) Experimental and Numerical Investigation of Chaotic Regions in the Triple Physical Pendulum. Special Issue of Nonlinear Dynamics 50 (4): 755-766.

[26] Awrejcewicz J, Supeş B, Lamarque C-H , Kudra G, Wasilewski G, Olejnik P (2008) Numerical and Experimental Study of Regular and Chaotic Motion of Triple Physical Pendulum. International journal of bifurcation and chaos 18 (10): 2883-2915.

[27] Awrejcewicz J, Petrov A G (2008) Nonlinear Oscillations of an Elastic Two Degrees-of-Freedom Pendulum. Nonlinear dynamics 53 (1-2): 19-30.

[28] Seyranian A P, Yabuno H, Tsumoto K (2005) Instability and Periodic Motion of a Physical Pendulum with a Vibrating Suspension Point (Theoretical and Experimental Approach). Doklady physics 50(9): 467-472.

[29] Seyranian A A, Seyranian A P (2006) The Stability of an Inverted Pendulum with a Vibrating Suspension Point. Journal of applied mathematics and mechanics 70: 754-761.

[30] Horton B, Sieber J, Thompson J M T, Wiercigroch M (2011) Dynamics of the Nearly Parametricpendulum. International journal of non-linear mechanics 46: 436-442.

[31] Blekhman I I (1954) Rotation of an Unbalanced Rotor Caused by Harmonic Oscillations of its Axis. Izv. AN SSSR, OTN 8: 79-94. (in Russian)

[32] Blekhman I I (1979) Vibrations in Engineering. A Handbook. Vol. 2. Vibrations of Nonlinear Mechanical Systems. Moscow: Mashinostroenie. (in Russian) 
[33] Blekhman I I (2000) Vibrational Mechanics. Nonlinear Dynamic Effects, General Approach, Applications. Singapore: World Scientific. 509 p.

[34] Akulenko L D (2001) Higher-order Averaging Schemes in the Theory of Non-linear Oscillations. Journal of applied mathematics and mechanics 65: 817-826.

[35] Trueba J L, Baltanáas J P, Sanjuáan M A F (2003) A Generalized Perturbed Pendulum. Chaos, solitons and fractals 15: 911-924.

[36] Fidlin A, Thomsen J J (2008) Non-Trivial Effects of High-Frequency Excitation for Strongly Damped Mechanical Systems. International journal of non-linear mechanics 43: 569-578.

[37] Caughey T K (1960) Hula-Hoop: an Example of Heteroparametric Excitation. American j. physics 28: 104-109.

[38] Horikawa T, Tsujioka Y (1987) Motion of Hula-Hoop and its Stability. Keio science and technology reports 40: 27-39.

[39] Wilson J F (1998) Parametric Spin Resonance for a Spinner with an Orbiting Pivot. Int. j. non-linear mech. 33: 189-200. 